Portland State University

PDXScholar

Summer 8-11-2017

\title{
Assessing a Fluorescence Spectroscopy Method for In-Situ Microbial Drinking Water Quality
}

Taylor Jeffery Sharpe

Portland State University

Follow this and additional works at: https://pdxscholar.library.pdx.edu/open_access_etds

Part of the Microbiology Commons, and the Water Resource Management Commons Let us know how access to this document benefits you.

Recommended Citation

Sharpe, Taylor Jeffery, "Assessing a Fluorescence Spectroscopy Method for In-Situ Microbial Drinking Water Quality" (2017). Dissertations and Theses. Paper 3838.

https://doi.org/10.15760/etd.5732

This Thesis is brought to you for free and open access. It has been accepted for inclusion in Dissertations and Theses by an authorized administrator of PDXScholar. Please contact us if we can make this document more accessible: pdxscholar@pdx.edu. 
Assessing a Fluorescence Spectroscopy Method for In-Situ Microbial Drinking Water Quality

by

Taylor Jeffery Sharpe

A thesis submitted in partial fulfillment of the requirements for the degree of

\author{
Master of Science \\ in \\ Mechanical Engineering
}

Thesis Committee:

Evan Thomas, Chair

Gerald Recktenwald

Derek Tretheway

Portland State University

2017 
(C) 2017 Taylor Jeffery Sharpe 


\begin{abstract}
Waterborne disease is a significant contributor to the global burden of disease, in particular among high-risk populations in developing nations. State-of-the-art methods for the enumeration of microbial pathogens in drinking water sources have important limitations, including high initial cost, 24-48 hour delays in results, high staffing and facility requirements, and training requirements which all become especially problematic in the developing nation context.

A number of alternative approaches to microbial water quality testing have been proposed, with the goal of decreasing the required testing time, decreasing overall costs, leveraging appropriate technology approaches, or improving sensitivity or specificity of the water quality testing method. One approach that may offer solutions to some of these limitations involves the deployment of sensor networks using fluorescent spectroscopy to detect intrinsic protein fluorescence in water samples as a proxy for microbial activity. In recent years, a number of researchers have found significant and meaningful correlations between indicator bacteria species and the protein fluorescence of drinking water samples. Additionally, advances in the semiconductor industry could be used to drive down the cost of such sensors. This technology may also be extensible to other water quality parameters, including dissolved organic matter or the presence of fluorescent pollutants.
\end{abstract}

In this thesis, a literature review describes the fundamentals of fluorescence spectroscopy, historical and recent work regarding the fluorescence of the amino acid tryptophan and associated bacterial fluorescence, possible mechanisms for this 
association, and potential applications of this technology for drinking water quality monitoring and waste water process control. Extensibility of the technology is also discussed.

Next, experimental methodology in reproduction of similar results is described. Samples were taken from seven (7) surface water sources and tested using membrane filtration and an off-the-shelf fluorescence spectrometer to help examine the association between the presence of indicator bacteria and the tryptophan fluorescence of the water sample. The results, showing an association of $\mathrm{R}^{2}=0.560$, are compared to the results of recent similar experiments.

Finally, two prototypes are described, including their design requirements and data from prototype testing. The results of the testing are briefly discussed, and next steps are outlined with the goal of developing a low-cost, in-situ microbial water quality sensor using fluorescence spectroscopy principles. 


\section{Dedication}

Dedicated to my parents, who taught me to start with the written word in my search for understanding; and who taught me to see even the biggest problems as surmountable and critical. And to my brothers and sisters, who have shown me a half-

dozen fascinating ways of interacting with and impacting the world. I'm proud of every one of you. 


\section{Acknowledgements}

The author wishes to extend heartfelt thanks to thesis advisor Evan Thomas, who provided that mythical combination of guidance and autonomy that fosters constrained but creative problem-solving. And to those who offered consistent guidance and new ideas in the SWEET Lab, especially Zdenek Zumr and Zak White, who were always interested and capable collaborators.

Many thanks to Mike Chuning for countless hours of training and support that provided the skills necessary to envision, design, and make useful parts.

Thanks to Gerald Recktenwald, Mark Weislogel, Derek Tretheway and Raúl Cal of the ME department for your design support in the early stages of this process. Your input was, and continues to be, invaluable. Thanks to ME faculty and staff for your continual encouragement and for providing the tools and skills necessary to approach difficult problems, parse and categorize them, and find creative solutions. And to the MELT Techs, whose dedication and enthusiasm enabled me to pivot from old responsibilities to new ones almost overnight.

And finally, thanks to my fellow students, especially Geng Qin, Joseph Ladd, Stone Rose, Christopher Patrick, and Julien Granich-Young. I owe so much to all of you for your brilliant insights, your unfailing senses of humor in the face of differential equations, and your constant support. 


\section{Table of Contents}

Abstract

Dedication $\quad$ iii

Acknowledgements $\quad$ iv

List of Tables $\quad$ vii

List of Figures $\quad$ viii

List of Abbreviations $\quad x$

Section 1: Scope of Thesis 1

Section 2: Background 3

2.1 Existing Primary Water Testing Techniques: Membrane Filtration and DST 7

2.2 Conceptual Alternative Testing Methods for the Developing World 9

2.3 Fluorescence Theory 11

2.4 Current State of Research 17

2.5 Possible Mechanisms for Bacterial Tryptophan Fluorescence 19

2.6 Extensibility of Fluorescent in-situ Sensors 21

Section 3: Experimental Methods \& Results 25

3.1 Sample Collection 25

3.2 Membrane Filtration Procedure 27

3.3 SMF4 Fluorimeter Procedure 30

3.4 Results 33 
4.1 Target Capabilities

4.2 Design Difficulty: Biofouling

4.3 Design Difficulty: Component Costs 


\section{List of Tables}

Table 1: Comparison of Existing Microbial Testing Technologies. Adapted from Bain et al. (2012) and from supplier literature.

Table 2: Common Aquatic Fluorophores and Naming Conventions. Adapted from Coble et al. (2014) and Hudson et al. (2007).

Table 3: Sequence and Location of Samples Taken

Table 4: Strength of Association between Microbial Contamination and TLF 36 


\section{List of Figures}

Figure 1: Simplified Perrin-Jablonski diagram showing energy transitions in luminescent processes

Figure 2: Tryptophan Molecule, one of three main fluorescent aromatic amino acids

Figure 3: Single-pulse Fluorescent Decay (left) and Sample Steady-state Fluorescent Intensity (right). Adapted from Lakowicz (2006).

Figure 4: Possible aquatic fluorescence sensing schemes. (a) Evanescent field loop probe (b) Simultaneous excitation/emission probe (c) Chemically-tapered nearfield sensor (d) 90-degree flow-through cell

Figure 5: Sampling Locations for Membrane Filtration and Tryptophan Fluorescence Analysis.

Figure 6: DelAgua water testing kit with Membrane Filtration Vacuum Chamber, MLSB growth medium, aluminum petri dishes, and sterile sample pad

Figure 7: Photographs of Membrane Filtration petri dishes with high colony count and some combined colonies (left) and low colony count (right). Red colonies are bacteria that cannot ferment lactose, and should not be included in TTC counts

Figure 8: SMF4 Fluorescent Spectrometer with Cuvette, Light Cover, and Custom Cuvette Holder/Cleaner

Figure 9: Two media bottles, S1 and S2, were tested together in the dual-incubation chamber using the same water samples to ensure similar results. 
Figure 10: Two water sample sources were diluted and tryptophan fluorescence readings were taken to ensure linear response from SMF4. Values on the $x$ axis are percentage concentrations of sample water to tap water, ranging from $0 \%$ to $1.2 \%$ concentration sample water.

Figure 11: TTC Colony Forming Unit Counts vs. SMF4 Tryptophan Fluorescence Readings. Overall linear correlation is $\mathbf{R}^{2}=0.56$

Figure 12: Exploded view of Repackaged Components from SMF-4

Figure 13: Simulated Geometries for Chang et al. (2006)

Figure 14: Simulated Geometries for Drost et al. (2014)

Figure 15: Comparison of linearity of signal response between SMF4 and Prototype 1 sampling chamber

Figure 16: Software Render of Prototype II with a Long UV LED Block (left) and a Short UV LED Block (right). All blocks can be replaced with others of varying geometries and with varying components

Figure 17: 3D Printed Prototype II Assembly, manufactured prior to machining 50

Figure 18: Rendered Drawing of Prototype II

Figure 19: Exploded View of Prototype II, showing top cap, exchangeable enclosures, emitter, detector, and optical equipment

Figure 20: Linear Relationship between Tryptophan Concentration and Prototype II Response (data from D. Wilson, S. Croshere, personal communication, 2017) 


\section{List of Abbreviations}

\begin{tabular}{|c|c|}
\hline Original Terminology & Abbreviation \\
\hline Colony-Forming Unit & CFU \\
\hline Defined Substrate Technology & DST \\
\hline Global Burden of Disease & GBD \\
\hline Membrane Laurel Sulphate Broth & MLSB \\
\hline Light-Emitting Diode & LED \\
\hline Photomultiplier & PMT \\
\hline Sustainable Development Goals & SDGs \\
\hline Target Product Profile & TPP \\
\hline Thermotolerant Coliform & TTC \\
\hline Tryptophan-Like Fluorescence & TLF \\
\hline Ultraviolet & UV \\
\hline United Nations International Children's Emergency Fund & UNICEF \\
\hline World Health Organization & WHO \\
\hline
\end{tabular}




\section{Section 1: Scope of Thesis}

Given the significant health burden associated with waterborne disease, there is a clear demand for sensor technologies that can monitor drinking water quality and enable a rapid response to contamination events, especially in the developing world. Existing technologies for the enumeration of microbial contamination in water samples have critical limitations, including the time required to generate results, high initial investment requirements, the need for trained lab technicians and sterile environments, and the need for a supply of perishable consumables (Bain et al., 2012).

In this thesis, fluorescence spectroscopy is described to introduce the concept of luminescence as a sensing tool. A literature review compares in-situ fluorescent instrumentation to alternative methods for microbial water quality testing. Existing technologies are briefly described, followed by a discussion of alternative proposed solutions and their relative strengths and weaknesses. These solutions are compared to a conceptual in-line, low-cost fluorescent bacterial sensor, with practical mechanisms for bacterial enumeration presented. Potential difficulties in the design and deployment of a fluorescent bacterial sensor are then addressed, along with possible solutions. The extensibility of the technology is discussed, including applications in the prevention of bioterrorism and in waste water treatment facilities.

Experimental methods and results are then described in which the output from a commercial, off-the-shelf tryptophan field fluorimeter (the SMF4 from Safe Training Systems) is compared to microbial colony counts from a field membrane filtration and incubation system (the DelAgua brand kit). These results are compared to results from 
the literature, and a call for standardization is given.

Two prototypes are then described. The first was designed and fabricated in order to repackage the optical elements from the SMF4 into a passive flow-cell cavity. Calculations for residence times within the sampling cavity are given, with estimates for the temporal resolution of the sensing unit based on multiple flow conditions. The second prototype is currently being evaluated for its sensitivity to tryptophan standards, using proprietary circuit boards, precision machined enclosure parts, and off-the-shelf optical components. The second prototype reflects the current progress of this research, and technology benchmarks and next steps are discussed. 


\section{Section 2: Background}

According to the World Health Organization, at least 1.8 billion people globally use drinking water from sources that are frequently contaminated with fecal material (Stedman, 2011). Diarrheal disease, despite being preventable through water quality testing and treatable through inexpensive means, is estimated to be responsible for 499,000 deaths of children under the age of five every year (GBD Collaborators et al., 2015). Waterborne disease is not limited to the developing world, with outbreaks of potentially-fatal bacteria commonly occurring in developed water sources, including in the United States (Beer, 2013 and Liang et al. 2006).

The demand for rapid, in-situ sensors for the detection of microbiological contamination in drinking water has been well-established in the literature (Stedmon et al. 2011, Bridgeman et al. 2013, Storey et al. 2011 and Paruch \& Maehlum 2012). Current detection methodologies for drinking water quality usually take the form of plate counts - in which bacteria are provided with nutrients, incubated for 16 to 24 hours, and then counted — or of defined substrate technology (DST), such as the Colilert method for testing for Escherichia coli (E. coli) contamination, allowing for higher specificity of results but still requiring at least 18 hours of incubation. Results from water samples arrive up to a day later than the actual sampling event. Because of this significant time requirement, detection of a contamination event prior to water's distribution to consumers is effectively nonexistent (Stedmon et al., 2011). Additionally, contamination events are stochastic and can occur quickly, making infrequent ad-hoc grab-sample approaches untenable as indicators of such events. 
UNICEF recently released a Target Product Profile (TPP) calling for technology that could enable real-time $E$. coli detection, citing many of these same concerns. The TPP highlights the significant costs to UNICEF and similar organizations, with approximately $\$ 450$ million in water testing procurement costs in 2013 (pg. 2). The proposal outlines potential demand for procurement within UNICEF, as well as external demand, citing the UN's Sustainable Development Goals (SDGs), which include goals around water quality targets, drinking water procurement, and community water resource management (pg. 2). The TPP tabulates minimum performance requirements for a suitable product, including battery-based operation, minimal processing requirements with preferably no need for reagent mixing or incubation, qualitative output based on quantifiable ranges of faecal contamination, the ability to sample a variety of water sources, and sensitivity and specificity goals equating to the ability to detect 10 ColonyForming Units (CFUs) per 100mL, with false positives and negatives below 10\%. Other product requirements are a detection time of less than 3 hours, a 2-year minimum lifespan, and portability (pg. 3-4).

One promising solution incorporates decades of research that have established the fluorescent properties of both individual bacteria species and water resources that contain viable bacteria. Recent efforts have shown strong correlations between the presence of tryptophan fluorescence and the presence of heterotrophic bacteria, E. coli, and total coliforms. As these bacteria are common indicator species for water contamination, significant increases in tryptophan fluorescence in a water distribution system may indicate contamination events. In contrast with standard water testing procedures, 
fluorescent readings can be obtained almost instantaneously. An added benefit is the relatively low cost of components given recent and dramatic decreases in the cost of semiconductor technology including deep-UV light emitting diodes (LEDs) and sensitive semiconductor photodiodes.

Fluorescent spectrometers consist of a narrow-bandwidth light source and a filtered light detector capable of emitting and detecting one or more wavelength pairs, corresponding to absorbed and emitted light. Some molecules exhibit fluorescent properties, meaning that excitation by one peak wavelength leads to emission of another through the excitation and subsequent relaxation of electrons in the molecule's structure. Fluorescent spectrometers use this effect to identify the presence and concentration of fluorescent molecules with high degrees of accuracy, and have been used in laboratory settings for the analysis of proteins and fluorescent elements for many decades. These molecules are referred to as fluorophores, and some may have multiple excitation and emission wavelengths. In the context of this research, a particularly important example of a fluorescent molecule is the aromatic amino acid tryptophan, thought to fluoresce due to the presence of a peripheral indole chain which contains weakly paired electrons. It should be noted that recent research calls into question the attribution of tryptophan fluorescence to the indole chain alone, based on fluorescent lifetime measurements: Alban (2014) found that the fluorescence properties of tryptophan and indole are not, in fact, identical. More work must be done to characterize what has become known as Tryptophan-Like Fluorescence (TLF), and can be expected in the coming years given the importance of this type of fluorescence in the analysis proteins in the laboratory setting. 
Beyond the protection of potable water, the applications of a low-cost, in-situ fluorescent sensor are numerous. The main application explored in this thesis is the monitoring of drinking water sources in impoverished communities, with a focus on the monitoring of improved water sources, especially distribution networks for groundwater sources. However, a variety of other fluorophores could be detected by a physicallyidentical system provided with a new set of optical components, which are generally produced in standardized electronics packages. This technique would allow for a single fluorescence platform to be used in the characterization of water samples with respect to a variety of properties interesting to environmental scientists, public health professionals, and even homeland security advisers.

Significant limitations and design difficulties exist in the use of in-situ fluorescence spectrometers, well synthesized by Conmey et al. (2014). First, all in-situ optical sensors are subjected to both biological fouling and mineral scaling, leading to sometimes-unpredictable changes in optical properties over the duration of the installation (pg. 213). Second, until recent years, expensive photomultipliers and highpower Xenon flash bulbs were required to generate a sufficient signal (pg. 201). Third, significant amplification and sophisticated signal processing techniques are required to obtain useful data from high-sensitivity detectors including both photomultipliers and newer semiconductor photodiodes, especially considering environmental confounders including temperature, particle scattering, oxygen bubbles, inner filtering effects (pg. 203-213)Finally, component degradation over the lifetime of the product requires either consistent re-calibration or some signal processing method to account for changes in 
baseline readings, necessitating some kind of calibration for data to be useful (pg. 208).

These difficulties are discussed in further detail in Section 4: Prototype Development.

\subsection{Existing Primary Water Testing Techniques: Membrane Filtration and DST}

In drinking water quality testing, the identification of indicator species including and especially E. coli is necessary for the qualification of a water resource as potable (Tallon et al., 2005). The microbes of interest are Thermotolerant Coliforms (TTC), a group of 4 primary bacterial species also including Klebisella, Enterobacter and Cirobacter. These coliforms are rod-shaped, gram negative, and share in common the ability to grow above $44^{\circ} \mathrm{C}$. They are facultatively anaerobic, capable of both respiration and fermentation, and are able to ferment lactose within 48 hours at $37^{\circ} \mathrm{C}$. Previously referred to as "faecal coliforms", TTC are present at high levels in the intestinal tracts of mammals and birds, showing minimal growth outside of this environment. WHO guidelines specify that drinking water should contain TTC levels below the threshold of 1 CFU per 100 milliliters of water (WHO, 2011).

Research indicates that the majority of TTCs are represented by various species of E. coli (Howard et al., 2003). E. coli presence, it is argued, is the best indicator for faecal contamination due to its ubiquity in mammalian intestinal tracts and its longevity following contamination events (Edberg et al., 2000). The presence of indicator species can indicate a failure in a water distribution system, which is especially important given the recent rise in the installation of dual-reticulation systems, which carry potable water to developed areas and recyclable or in the opposite direction. A failure between these lines can cause immediate contamination and lead to severe health risks (Hambly et al. 
2010). Other contamination events might include rupturing sewage lines, groundwater leaching, or flooding events. Paruch \& Mauhlum (2012) point out that while the presence of E. coli may not directly indicate pathogenic organisms, it is "The most accurate indicator of faecal pollution..." (pg. 141). They also categorize a set of five strains of $E$. coli as particularly important pathogenic agents associated with poor hygienic and sanitary conditions - Enterotoxigenic, Enteropathogenic, Enteroinvasive, Enteroaggregative and Diffusely Adherent E. coli (pg. 141). Unlike the strains most commonly appearing in publicized US events, which are Veroctotoxic E. coli, these strains are generally water-borne rather than transferred between produce and the human digestive tract.

Two primary techniques used to quantify either TTCs or E. coli directly are membrane filtration and DST. Membrane filtration makes use of a vacuum chamber which draws water across a semi-permeable membrane, adhering bacterial cells to the membrane. This is then placed in a plate with a broth of ideal nutrients, and incubated for 18-24 hours until each cell has multiplied to the point where it can be seen with the naked eye, predicated on the assumption that each colony has been formed by one individual original cell. Often, the broth contains compounds which change color selectively at the fermentation of lactate, helping to reduce false positives by identifying actual coliforms.

DST allows for simultaneous quantification of total coliforms and $E$. coli through the use of compounds that become fluorescent upon contact with $\beta$-glucuronidase, an enzyme specific to $E$. coli among coliforms. Following a similar incubation period, a handheld UV lamp can be used to identify and count colonies. A number of similar 
techniques exist on the market, at a variety of initial and consumable price points, and are shown in Table 1, drawn from a number of sources including (Bain, 2012) and pricing literature from the websites of distributors, based on costs from 2015. It is clear that costs for microbial water quality testing are high, and are therefore not feasible for widespread use in the developing world.

Table 1: Comparison of Existing Microbial Testing Technologies. Adapted from Bain et al. (2012) and

\begin{tabular}{|l|c|c|c|c|c|c|c|}
\hline Technology & Petrifilm & Colilert-18 & $\begin{array}{c}\text { Hydrogen } \\
\text { Sulphide }\end{array}$ & $\begin{array}{c}\text { Total } \\
\text { Coliform }\end{array}$ & $\begin{array}{c}\text { Colilert/Quanti- } \\
\text { Tray }\end{array}$ & $\begin{array}{c}\text { Membrane } \\
\text { Filtration }\end{array}$ & $\begin{array}{c}\text { Theoretical } \\
\text { Fluorimeter }\end{array}$ \\
\hline Time to Result (hr) & 24 & 18 & 48 & 35 & 21 & 24 & 0.1 \\
\hline Precision (0-3) & 3 & 3 & 0 & 0 & 3 & 3 & 0 \\
\hline Cost per Test (\$) & 1.3 & 5 & 1.325 & 1 & 5.5 & 0.5 & 0.01 \\
\hline Initial Cost (\$) & 100 & 100 & 0 & 50 & 4100 & 2500 & 1500 \\
\hline
\end{tabular}

In the resource constrained environments, difficulties status quo water quality testing techniques fall into four broad categories. First, they require highly-trained lab technicians and sterile environments. Second, they generally require 16 to 48 hours of time between samples being taken and results being generated. Third, they generally require a constant supply of consumables and electrical power, which can prove difficult in many developing world or disaster relief settings. Finally, they come at high initial cost, requiring the purchase of incubation and sterilization technology.

\subsection{Conceptual Alternative Testing Methods for the Developing World}

Candidate water quality testing solutions are reviewed in Bain et al. (2012). In general, little can be done to replace membrane filtration or standard assaying techniques, with the focus instead being placed on replacing expensive equipment with more appropriate, low-cost local solutions - for example, replacing a laboratory incubator with an egg incubator or replacing an autoclave with a pressure cooker or chemical 
disinfectant. Other suggestions include replacing de-ionized water with boiled water or using an autoclave if already available; replacing electrical membrane filtration apparatuses with hand pumped portable versions; and allowing samples to be transported or stored at room temperature to offset refrigeration costs.

One solution addressed elsewhere in the literature (Brown et al., 2011) is ambient incubation. In tropical environments, it may be capable to offset the initial cost of an incubator by performing incubations at ambient temperatures; however, research suggests that while this approach is viable in terms of accuracy, it requires significantly increased incubation times, therefore moving even further from the ideal solution, which would be quick, accurate, and broadly applicable at low costs.

Another option is to replace quantitative analyses with presence-absence tests, specifically the hydrogen sulphide $\left(\mathrm{H}_{2} \mathrm{~S}\right)$ presence-absence test, which is available at a lower cost, requires a lower level of training for users, can be performed at ambient temperatures, and is emerging as an alternative to quantitative methods in developing countries and in emergency settings. Presence of $\mathrm{H}_{2} \mathrm{~S}$-producing bacteria has been proposed as a proxy for faecal contamination, since there appears to be an association between presence of coliforms and these hydrogen sulphide producing bacteria; in addition, certain enteric bacteria including Salmonella also produce the gas (Manja et Al, 1982). However, research regarding the association between $\mathrm{H}_{2} \mathrm{~S}$ tests and the presence of common indicator organisms is split: while Khush et al. (2013) found a strong relationship between $\mathrm{H}_{2} \mathrm{~S}$ results and total coliform counts in a large prospective cohort study in rural India $\left(\mathrm{R}^{2}=0.87\right)$, a systematic review and meta-analysis by Wright et al. 
(2012) found high variability in the specificity of the test, unexplained by differences in procedures, sample types or study quality.

\subsection{Fluorescence Theory}

Fluorescence is the emission of photons following an atom or molecule's excitation by other photons. An excitation wavelength is responsible for the excitation of the fluorophore's electrons to higher energy states. The electron quickly drops to the lowest excited vibrational energy state through the process of internal conversion, occurring between femtoseconds and picoseconds. Fluorescence then occurs as the electron drops to some base energy state, emitting a photon. The time between internal conversion and fluorescence is dependent upon the fluorophore in question as well as environmental factors and polarization.

Because some degree of internal energy loss occurs following excitation and preceding the emission of the new photon, the emitted light is of lower energy and therefore is emitted at a longer wavelength, an observation referred to as the Stoke shift. The overall decrease in this energy is related to both the emission of the photon (radiative decay) and to internal conversion processes including relaxation and collisional quenching (nonradiative decay).

Below, a simple Perrin-Jablonski diagram shows the energy paths describing fluorescence and phosphorescence. Figure 1 shows a molecule that begins at some base energy state $\mathrm{S}_{0}$ excited through absorption to a higher (singlet) energy state, $\mathrm{S}_{1}$. A number of subsequent potential energy transformation mechanisms can now act on the excited molecule; in addition to fluorescence, which occurs within nanoseconds, some energy is 
lost due to intersystem crossing, as well as through radiative decay from both the excited energy state and the lower $\mathrm{T}_{1}$ triplet energy state. Note that other de-excitation pathways exist, including, for example, direct interactions with surrounding molecules.

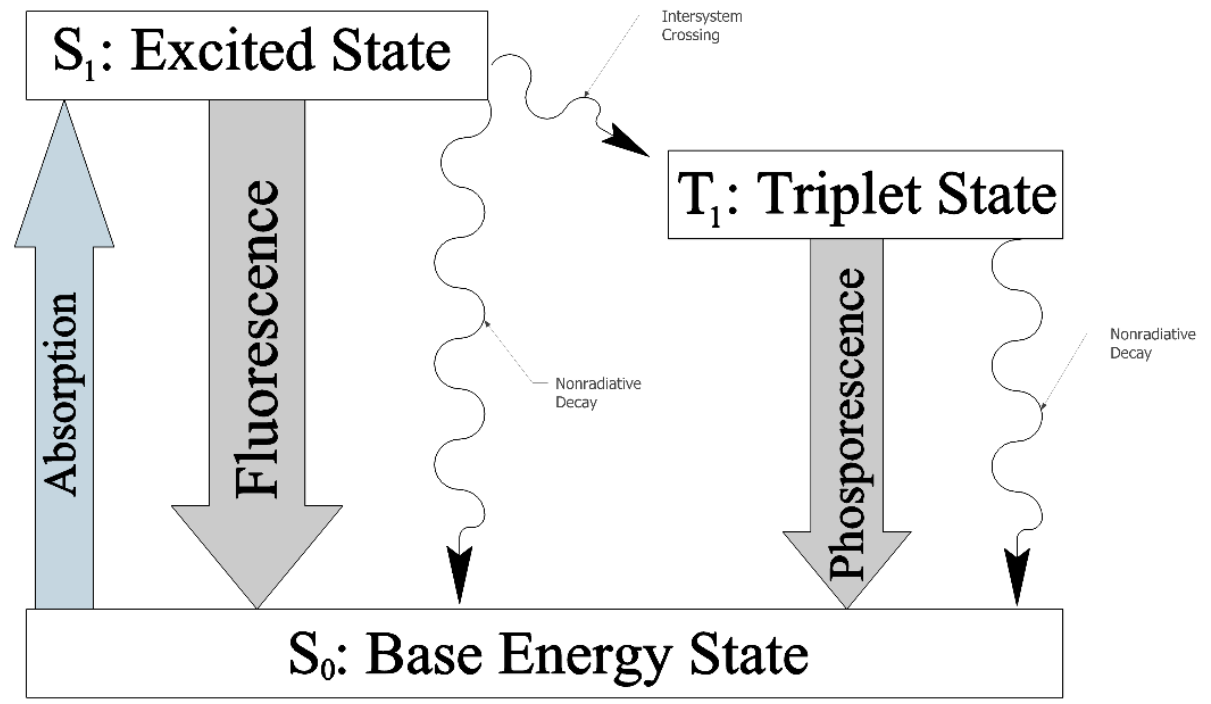

Figure 1: Simplified Perrin-Jablonski diagram showing energy transitions in luminescent processes

Of special importance in the measurement of fluorophores in a sample is the quantum yield, the fraction of excited molecules that fluoresce due to an energy transformation to their base energy state, defined as,

$$
\Phi_{F}=\frac{k_{r}^{S}}{k_{r}^{S}+k_{n r}^{S}}
$$

where $k_{r}^{S}$ is the rate constant for radiative deactivation through fluorescence and $k_{n r}^{S}$ is the rate constant for nonradiative deactivation due to the process of intersystem crossing in addition to other energy decay processes outside of the emission of light.

The time in which a particular fluorophore falls from its excited state to its base energy state is called the "decay time", $\tau$. Note that this is an average amount of time spent in the excited state, rather than some intrinsic property of the fluorophore. 
Fluorescent lifetimes are generally on the scale of $10 \mathrm{~ns}$.

Most proteins fluoresce when stimulated by light in the UV region. Three aromatic amino acids are thought to be responsible for protein fluorescence: tryptophan, tyrosine, and phenylalanine. Of these, tryptophan, whose chemical structure is shown in Figure 2, is the most highly fluorescent (Sauer et al., 2011). Tryptophan has a quantum yield of between 0.1 and 0.5 , has the highest absorption coefficient of the three fluorescent amino acids, and has the longest emission wavelength.

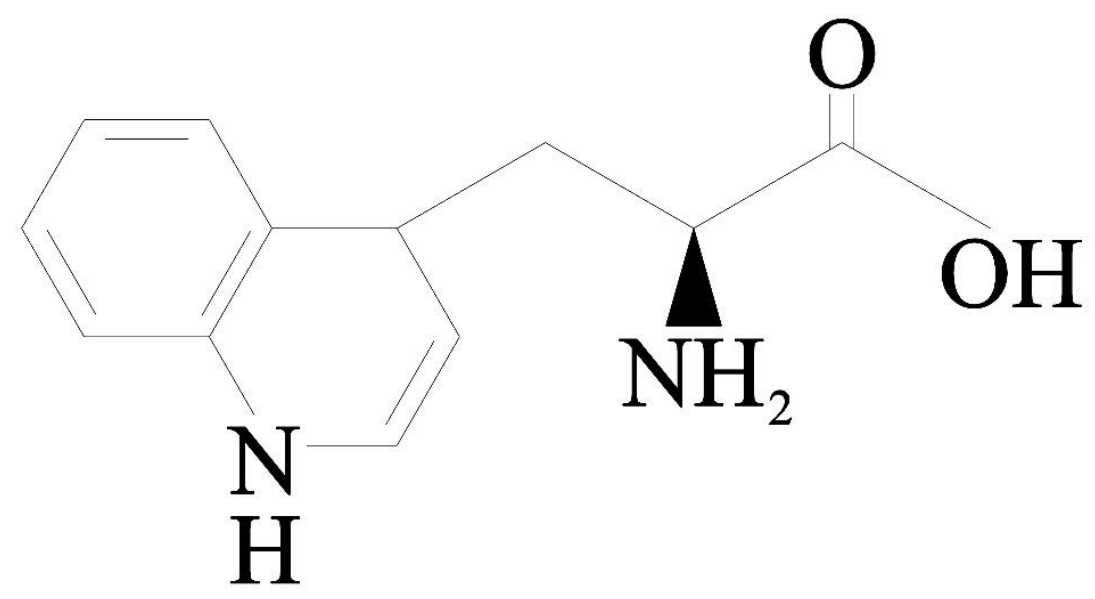

Figure 2: Tryptophan Molecule, one of three main fluorescent aromatic amino acids

Contrary to the simplified model in Figure 1, protein fluorescence results from a complex arrangement of amino acids folded into place in a protein; organic molecules' fluorescent properties are thought to be the result of the overlapping fluorescent behaviors of the amino acids that make up their proteins. At times, proteins can be characterized and protein folding mechanisms can be examined by measuring fluorescent properties of these three components. Changes in protein fluorescence can also be used as a proxy for changes to the proteins. Tryptophan is especially useful as a reporter for conformation changes in proteins, since a number of protein changes of great interest to 
the chemist or biologist can result in predictable changes in tryptophan fluorescence in a sample. For example, oxygen concentrations in solution can be measured by measuring the change in the fluorescence lifetime of certain fluorophores whose signal is selectively quenched by oxygen.

Intrinsic fluorescence of low-turbidity samples can be measured using a steadystate fluorescence spectrometer: the light emitter is powered on and brought to a constant-intensity state, the detector is used to take a sequence of readings, which are converted into an electrical signal and averaged for subsequent interpretation.

The relationship between molecular fluorescence and the steady-state output from a fluorimeter holding an emission source at a steady output level is shown in Figure 3. A single pulse of light results in the rapid decay of the sample's fluorescence, where a steady source of excitation light results in one average emission intensity. As detailed in Lakowicz (2006), steady state signals from a fluorimeter are "... simply an average of the time-resolved phenomena over the intensity decay of the sample." (pg. 14).
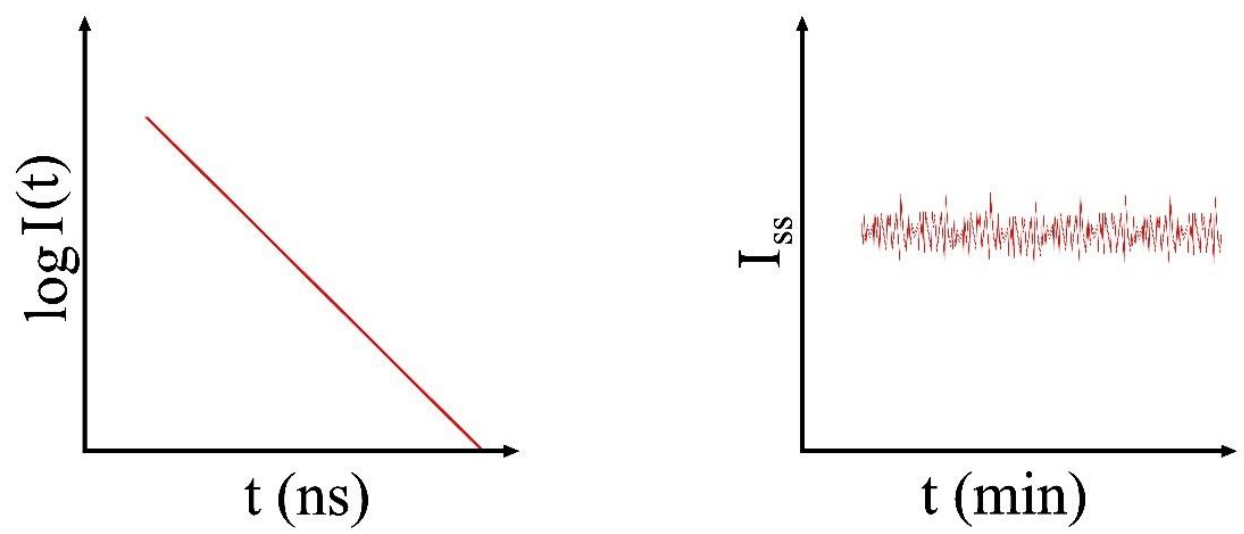

Figure 3: Single-pulse Fluorescent Decay (left) and Sample Steady-state Fluorescent Intensity (right). Adapted from Lakowicz (2006). 
The steady-state intensity of the sample is proportional to the fluorescent lifetime of the molecule. With the value $\tau$ equal to the fluorescent lifetime of the molecule and the effective intensity value $\mathrm{I}_{0}$ resulting from the concentration of the fluorophore in the sample, the properties of the instrument, and some chemical properties of the sample fluid, the steady-state spectral intensity is given by,

$$
I_{S S}=\int_{0}^{\infty} I_{0} e^{-\frac{t}{\tau}} d t=I_{0} \tau
$$

Steady-state fluorescence can be understood as the state at which fluorescent intensity of a large number of fluorophores has balanced out the effects of fluorescent emission and absorption - that is, the exponential decay and growth of the fluorescent signal of each molecular fluorophore is balanced out by the reverse processes of the other fluorophores in the solution. Therefore, if the intensity of the light source can be kept constant, steady-state fluorescent emission intensity should quickly stabilize. However, it should be noted that a significant quantity of information is lost in this conversion to steady state: for example, many molecules including tryptophan actually exhibit multiple fluorescent lifetimes, which can change based on environmental conditions, but the extra insights one could glean from the change in these peaks are all lost when only steadystate fluorescence is measured.

A variety of optical sensing schemes, including configurations in which the water is sampled with beams of light propagating through windows and directly through the sample, or configurations in which fiber optics probes are used to deliver and collect excitation and emission light either directly or via evanescent field readings, have been 
explored in the literature. Figure 4 shows a number of potential sensing schemes, some of which would incorporate immunoassaying techniques in which antigens against particular bacteria species could be immobilized on fiber-optic probes in order to capture them and enable near-field sensing. These techniques are expensive and highly-specific, leading most researchers in the field to focus on the use of simple cuvette-based or flowthrough sampling schemes in order to spare expense and manufacturing difficulties.

For the purpose of this research, sensing scheme (d) is the one used, both in the case of the field spectrometer used and in the case of both prototypes developed. A 90degree optical cell is chosen in order to avoid the cost and complexity of fiber optic signal processing, cladding removal, and immunoassaying.

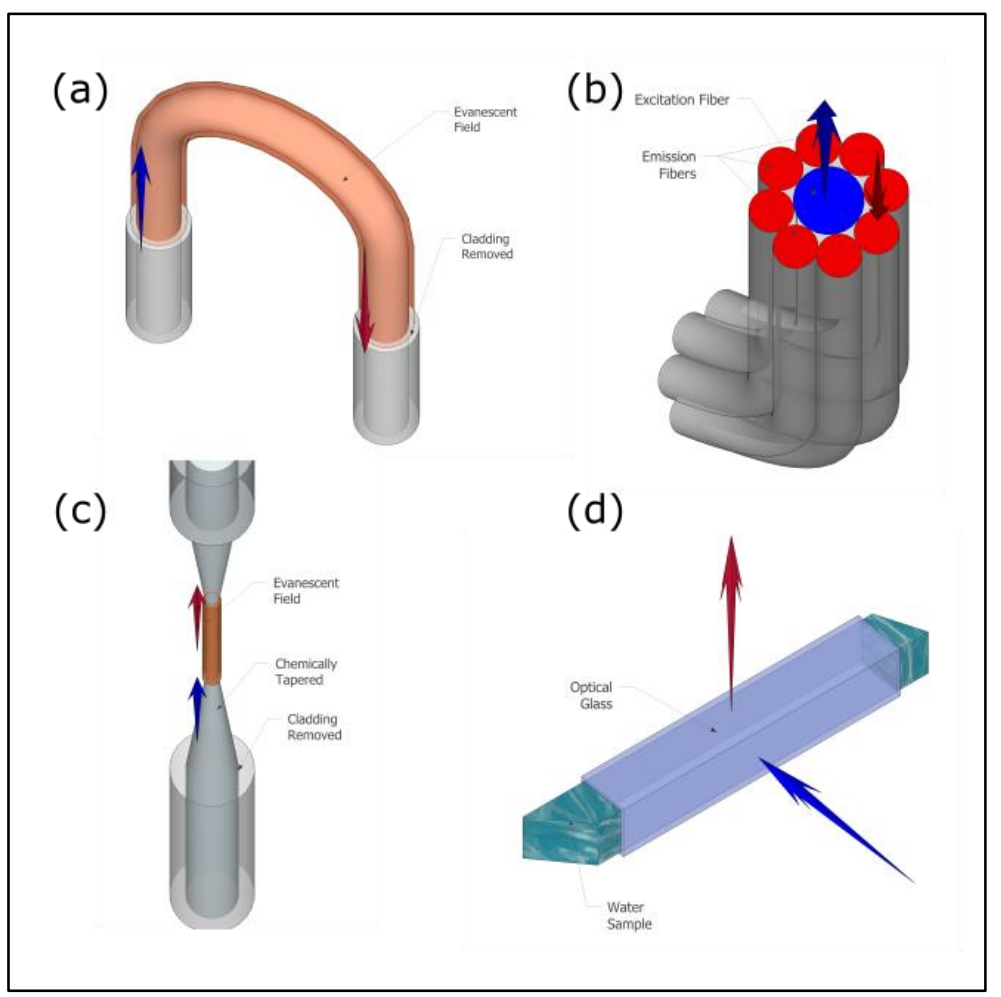

Figure 4: Possible aquatic fluorescence sensing schemes. (a) Evanescent field loop probe (b) Simultaneous excitation/emission probe (c) Chemically-tapered near-field sensor (d) 90-degree flow-through cell

It should be noted that proper fluorescent measurements are hindered by a number 
of factors, especially by mechanical and optical properties which can change the wavelength of either excitation or emission light. This includes the emitter, which may show changes in both peak wavelength and intensity over its lifetime; the detector, which might show a difference in wavelength-based response over its lifetime; and any optical elements between these two. This often necessitates the use of calibrated correction factors for both wavelengths and intensities, quickly multiplying the complexity of the instrument (Valeur and Berberan-Santos, 2013). Often, a reference chamber is included in order to allow for adjustments to be made over the lifetime of the machine. The reference chamber contains a fluorescent compound expected to change very little in its fluorescent response over the lifetime of the machine, functioning as a baseline reading from which back-subtraction can be performed.

Changes to the composition of the sample can have significant effects on the fluorescent signal even if they do not inherently change the concentration of the fluorophore in the sample. Some of these difficulties will be discussed in a later section.

\subsection{Current State of Research}

Research has recently been conducted with similar goals in terms of both bacterial detection (Ferreira et al., 2001 and Bharadwaj et al., 2011) and in-situ fluorescent analysis (King et al., 2000 and Van Bergen et al, 2000). However, these solutions require extensive and precise modification of current technology, making them unsuitable for low-cost, large-scale deployment and autonomous operation. By shifting the research focus from sensitive analyte detection to an order-of-magnitude scale in which a sudden contamination event can trigger an alarm, instruments could be produced using simpler 
technology and on a much wider scale. Furthermore, although research has been conducted to show the ability of field fluorimeters to detect water contamination to the 100CFU/100ml level (Cumberland, 2012 and Tedetti, 2013), these techniques still require that resources be sampled by hand, excluding remote monitoring and requiring the presence of trained lab technicians. It is worth noting that this contamination level of TTCs puts this type of test on par with hydrogen sulphide presence/absence tests (Yang et al., 2013). An in-situ, on-line device that requires minimal maintenance would simultaneously increase the rapidity and decrease the required manpower involved in water quality testing. These potential sensing schemes would incorporate immunoassaying techniques in which antigens against particular bacteria species could be immobilized on fiber-optic probes in order to capture bacteria, enabling near-field sensing. These techniques are expensive and highly-specific, reinforcing the conclusion that a simple flow-through cell might be the best option for an inexpensive device that can be used in a wide variety of water resources and incorporated into a sensor grid.

A number of researchers have analyzed the ability of fluorescent spectrometers to qualify water resources, including the use of tryptophan fluorescence as a proxy for bacterial activity. Cumberland et al. (2012) found strong correlations between tryptophan and the colony counts of a variety of indicator species, including E. coli. More recently, their dataset was expanded and combined with others to find an overall correlation of $r=$ 0.74 (Baker et al., 2015).

A second approach, based not on quantitative plate counts but on binary presenceabsence of TTCs, found that mean TLF concentration was significantly associated with 
the results of presence-absence Colilert tests in 150 samples of drinking water supplies in rural India based on a logistics-regression model ( $\mathrm{p}<0.001)$ (Sorenson et Al, 2015). The same study found that TLF could be considered “...a less transient faecal indicator in groundwater.” (pg. 44), providing a conservative estimate of site risk and overcoming issues associated with sampling frequency and seasonal variation. This suggests that spot samples of TLF may have the advantage of showing lower seasonal variation than traditional TTC plate counts, meaning that the time of sampling might play less of a role in the classification of the drinking water source as unsafe. This is a particularly important point given recent findings that even "improved" water sources are often faecally contaminated, a gap in information partially due to infrequent sampling of water resources (Bain et al., 2014), and that recent research indicates the failure of low-cost Sanitary Risk Scores to predict presence-absence of TTCs (Snoad et al., 2017).

Other elements require more research before the technology is mature enough for wide-scale deployment: automated data analysis is a vital requirement (Henderson et al., 2009), as well as analytical techniques to overcome physical difficulties in in-situ monitoring, including biofouling, flowrate concentration effects, and sensor network configuration optimization.

\subsection{Possible Mechanisms for Bacterial Tryptophan Fluorescence}

The source of TLF as associated with microbial water quality is not well understood mechanistically. It is still unclear whether this association is a result of the expected relationship between microbes and their bioavailable substrate, a result of microbial metabolic processes such as the cleaving of indole by E. coli, or a combination 
of these factors (Hudson et al., 2007).

Decades ago, researchers were already examining fluorescence as a tool for bacteria detection and even identification. Dalterio et al. (1986) were able to establish fluorescent decay spectrums for a number of bacterial species, including E. coli. The techniques applied were significantly more complicated than sampling intrinsic fluorescence, requiring electronics and signal processing capabilities that are an order of magnitude more precise and expensive than those used in standard tryptophan fluorometers. While their findings could be incorporated into very precise, expensive immunoassay sensors, it is unlikely that direct bacterial fluorescence is responsible for the high quantities of tryptophan fluorescence present in water resources which have been contaminated with waste water such as the samples examined by Bridgeman et al. (2013). Instead, they suggest that since tryptophan is both a substrate for and a byproduct of bacterial activity, it may be more accurately considered a proxy for the presence of bacterial organisms.

One possible mechanism explaining the association between TTCs and TLF is the accumulation of indole as both an intercellular (Lee \& Lee, 2010) and extracellular (Wang et al., 2001) signaling molecule for E. coli, or a variety of proteins accumulating as a possible defense mechanism during survival processes (Arana et al., 2004). Indole has recently been found to modulate a number of processes in bacterial species, including motility, biofilm formation, and virulence (Li \& Young, 2012, references therein). Indole has also been shown to protect $E$. coli from a variety of antibiotics (Lee et al., 2010). It may be that $E$. coli's fluorescent signature is due to cellular or extracellular accumulation 
of fluorescent material, since indole's intrinsic fluorescent signature is similar to that of tryptophan. Further research is needed to understand the exact mechanisms behind this relationship, but high degrees of success in correlating tryptophan fluorescence and bacterial presence can be utilized now. Deployment of sensors that use this association may actively contribute to this research as their use is increased in scope and scale.

\subsection{Extensibility of Fluorescent in-situ Sensors}

Deployment of fluorescent sensors in water distribution networks or in areas of environmental concern could drive down monitoring costs and increase the potential for real-time water quality characterization with respect to microbial contamination and zoologic waste management, a field in which fate, transport and health effects are not yet well understood (Dufour \& Bartram, 2012).

In addition to its promising capabilities for application in the fields of sanitation and public health, successful development of an in-situ, telemetry-enabled and low-cost fluorimeter could have significant impact on a variety of research fields. The detection of tryptophan fluorescence is applicable in the study of aquatic organic matter transport (Baker et al. 2004, Yamashita et al. 2003, and Hudson et al. 2007) and biological oxygen demand in rivers (Bridgeman et Al, 2013). Such an instrument also has implications regarding energy use and carbon emissions, since current testing methodologies during wastewater treatment often necessitate increased energy use in the aeration process by an estimated unnecessary 40\% (Bridgeman et al, 2013), and also involve labor-intensive testing processes. Real-time, autonomous water quality monitoring during the treatment process could decrease both energy use and overall costs in waste water treatment plants, 
while monitoring of effluent could provide feedback on the quality of the treatment process.

A second application involves rapid deployment in areas recently affected by natural disasters or infrastructure failure. The ability to quickly identify water infrastructure failure could prove invaluable in the real-time management of health risks on the ground. Another potential advantage of an inexpensive, simplified fluorescence sensor is its application to other fluorophore detection. It may be possible to design the device in such a way that UV LED light sources can be exchanged, along with an exchange of optical filters, to quickly (potentially on-site) adapt to a different target fluorophore. Therefore, the potential exists for real-time characterization of a variety of analytes in a variety of water resources, including pumps, reservoirs, rivers, streams, and lakes. Multiple sensors could be used in tandem to more fully characterize water sources. A third application is a response to recent concerns regarding bioterrorism.

In-situ fluorescent spectrometers can be used to measure the concentrations of a number of fluorophores in aqueous solution, including humic-like components, proteinlike components, chlorophyll, and leachant agents. These compounds are of significant interest to environmental scientists and engineers. Individual paired LED excitation sources and photodiode detectors and filters could be used to detect any of these fluorophores, likely with little or no modification of the fluorimeter's exterior enclosure. It has become common to refer to these compounds or mixtures of compounds by a "peak" name, for example, Peak T for tryptophan-like fluorescence (which is subsequently broken into Peak T1 and Peak T2 for different excitation-emission pairs). 
Table 2 shows a combined list of fluorophores of interest drawn from Coble et Al. (2014) and Hudson et Al. (2007) with references therein.

Table 2: Common Aquatic Fluorophores and Naming Conventions. Adapted from Coble et al. (2014) and Hudson et al. (2007).

\begin{tabular}{|l|c|c|c|}
\hline \multicolumn{1}{|c|}{ Fluorophore } & Peak Name & Excitation $\lambda$ & Emission $\lambda$ \\
\hline Humic-like & $\mathrm{A}$ & 240 & $350-400$ \\
\hline Humic-like & $\mathrm{C}$ & 260 & $400-460$ \\
\hline Humic-like & $\mathrm{C}+$ & 250 & $470-504$ \\
\hline Marine Humic-like & $\mathrm{M}$ & $290-310$ & $370-420$ \\
\hline Tyrosine-like & $\mathrm{B}$ & 230 & 305 \\
\hline Tryptophan-like & $\mathrm{T} 1$ & $225-237$ & $340-381$ \\
\hline Tryptophan-like & $\mathrm{T} 2$ & 275 & 340 \\
\hline Chlorophyll a & $\mathrm{n} / \mathrm{a}$ & 435 & 659 \\
\hline Chlorophyll b & $\mathrm{n} / \mathrm{a}$ & $220-230$ & $340-370$ \\
\hline Naphthalene leachant & $\mathrm{n} / \mathrm{a}$ & $220-230$ & $340-370$ \\
\hline Napthalene whitening agent & $\mathrm{n} / \mathrm{a}$ & $260,260,400$ & $430,540,460$ \\
\hline Pigment-like & $\mathrm{P}$ & 398 & 660 \\
\hline Photobleached & $\mathrm{H}, \mathrm{P} 4$ & 230 & $275-350$ \\
\hline
\end{tabular}

Additional sensing capabilities can be attained by combining two fluorophores, for example by sampling multiple fluorescent components in tandem, as explored by Stedmon et al. (2011), who were able to characterize the introduction of wastewater to drinking water supplies with a high degree of accuracy by monitoring the change in some components and the stationarity of others.

The extensibility of on-line water quality sensors applies also to the field of drinking water security, in reference to both intentional and unintentional contamination of drinking water sources. Janke et al. (2006) used a computer simulation to perform a risk analysis on three conventional water monitoring strategies with the goal of reducing health effects following an intentional biological or chemical agent release into a drinking water distribution system. 24 hour, 48 hour, and weekly sampling strategies were compared to the use of sensors that are "ideal (real-time, inline, contaminant specific)" 
(pg. 311). Their model examined pathways from an initial contamination point to simulated consumers, in order to quantify the health impacts of the contamination given a rapid-response sensing scheme. Their findings indicated that a smaller number of monitored nodes could offer significant preventative power compared to conventional monitoring techniques, even daily ones. In the case of chemical contaminants, for example, they found that the use of real-time monitoring of only a small number of nodes could “...reduce fatalities from 17 to $38 \%$ and maximum (worst case) fatalities by approximately 35\%" (pg. 312). Using similar methodology, Murray et al. (2006) find that in a simulated water contamination event, over half of all exposure occurs within the first 20 hours.

Jain et al. (2014) make some observations about the current status of on-line water contaminant sensors. Between Dec. 2005 and Jan. 2011, 80 events of public threats to water supplies were identified worldwide, often in the form of planned attacks that were prevented from being carried out. Since these data come from news reports, it is possible that the number of planned threats was actually higher. In response, the U.S. EPA has adopted a "Defense-in-Depth Approach" as a strategy for protective water management, including the adoption of contamination detection technologies and deployment strategies to manage detected attacks. The authors also acknowledge dual-use benefits, saying that specific attention should be paid to system that can add a second benefit along with primary water security - for example, real-time water quality sensors are not only earlywarning systems, but also provide useful and actionable information regarding baseline water quality parameters and water quality changes related to non-attack processes. 


\section{Section 3: Experimental Methods \& Results}

In order to help assess the suitability of a tryptophan fluorescence spectrometer as a tool for drinking water quality monitoring, surface water samples were tested using conventional TTC enumeration methods and simultaneously analyzed for TLF. Water samples were collected from seven locations along the Willamette River and in two other locations in Portland, Oregon. Water samples were analyzed in the Portland State SWEET Lab. On the day of collection, water samples were processed by membrane filtration and incubated at $44^{\circ} \mathrm{C}$, with control samples to identify any sample contamination, and were counted the following day after 18 hours of incubation. Tryptophan fluorescence readings were taken from the same samples during each membrane filtration event. Bacterial growth medium was tested on one occasion to ensure proper sterilization, and the output of the fluorimeter was tested with samples of varying concentration and turbidity to ensure that its readings corresponded to tryptophan concentration and not to other factors. The sample data was then compiled in order to examine the strength of the association between TLF and TTC colony counts, resulting in a $\log -\log$ linear relationship of $\mathrm{R}^{2}=0.56$.

\subsection{Sample Collection}

Figure 5 shows the seven locations from which water samples were taken, shown in red. Samples were collected in groups of 2 to 4 and transported to the lab for testing within one hour. The location of the lab is shown in blue. 


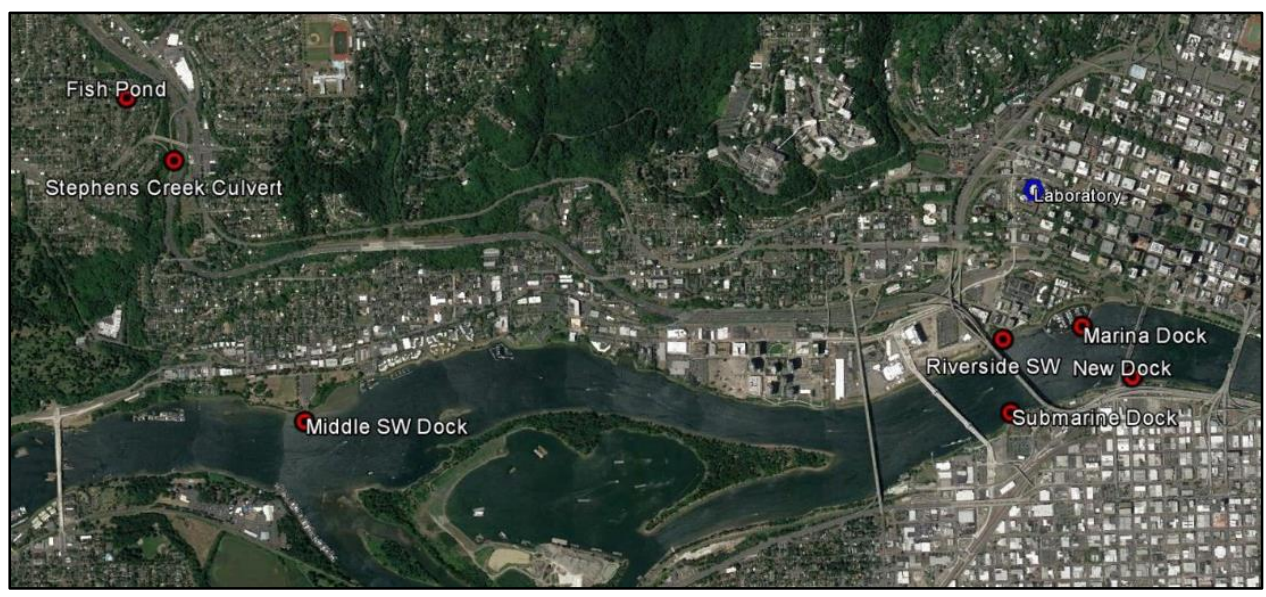

Figure 5: Sampling Locations for Membrane Filtration and Tryptophan Fluorescence Analysis.

Following a number of practice datasets in which the sampling methodology was refined, a total of 12 sampling events were included in the analysis. The number of total data points is 31 , of which 3 were removed due to sampling errors or very high microbe counts. Table 3 shows the sequence in which samples used in the final analysis were collected from these sources.

\begin{tabular}{|c|c|c|c|c|c|c|c|}
\hline \multirow[b]{2}{*}{$\begin{array}{c}\text { Sample } \\
\text { Number }\end{array}$} & \multicolumn{7}{|c|}{ Sample Location } \\
\hline & $\begin{array}{c}\text { Marina } \\
\text { Dock }\end{array}$ & New Dock & $\begin{array}{c}\text { Riverside } \\
\text { SW }\end{array}$ & $\begin{array}{c}\text { Submarine } \\
\text { Dock }\end{array}$ & Middle SW & Culvert & Fish Pond \\
\hline 1 & $\mathrm{x}$ & & $\mathrm{x}$ & & $\mathrm{x}$ & & \\
\hline 2 & $x$ & $\mathrm{x}$ & $\mathrm{x}$ & $x$ & & & \\
\hline 3 & $x$ & $x$ & & $x$ & $x$ & & \\
\hline 4 & $\mathrm{x}$ & $\mathrm{x}$ & & $\mathrm{x}$ & & & $x$ \\
\hline 5 & $\mathrm{x}$ & $\mathrm{x}$ & & $x$ & $\mathrm{x}$ & & \\
\hline 6 & $x$ & $\mathrm{x}$ & & $x$ & & & $x$ \\
\hline 7 & & & & & & $x$ & \\
\hline 8 & & & & & & $\mathrm{x}$ & $x$ \\
\hline 9 & $\mathrm{X}$ & & & & & $\mathrm{X}$ & $x$ \\
\hline 10 & & & & & & $x$ & $x$ \\
\hline
\end{tabular}

Samples were collected in clean, plastic bottles. Bottles were thoroughly cleaned and rinsed with DI water following each sample, and stored in a fume hood with the caps secure. At the interface between surface water and sample bottles, a stainless-steel cup 
was used to collect samples in keeping with the DelAgua Kit instruction manual. In all river samples, samples were collected at a depth of at least 1 foot. At the fish pond and culvert sampling locations, this was not possible given the geometries of the waterways; fish pond samples were collected just below the surface of the water, and culvert samples were collected at the outflow of a pipe.

Samples were transported in a side bag by bicycle, and were sampled immediately upon returning to the laboratory. The maximum time between a sample's collection and its membrane filtration and fluorimeter testing was 2 hours.

\subsection{Membrane Filtration Procedure}

Membrane filtration was used to measure the number of viable TTC colonies in water samples. Membrane filtration measurements were carried out using a dualincubator Oxfam-DelAgua Water Testing Kit (Figure 6) according to training provided by DelAgua staff and according to the DelAgua Kit instruction manual. Coliform growth plates, sterilized media pads, and a vacuum chamber with 0.45 micrometer pore size were acquired from DelAgua, Inc. Membrane Laurate Sulfate Broth (MLSB) powder was acquired through HiMedia India and prepared and sterilized per DelAgua's instructions. 


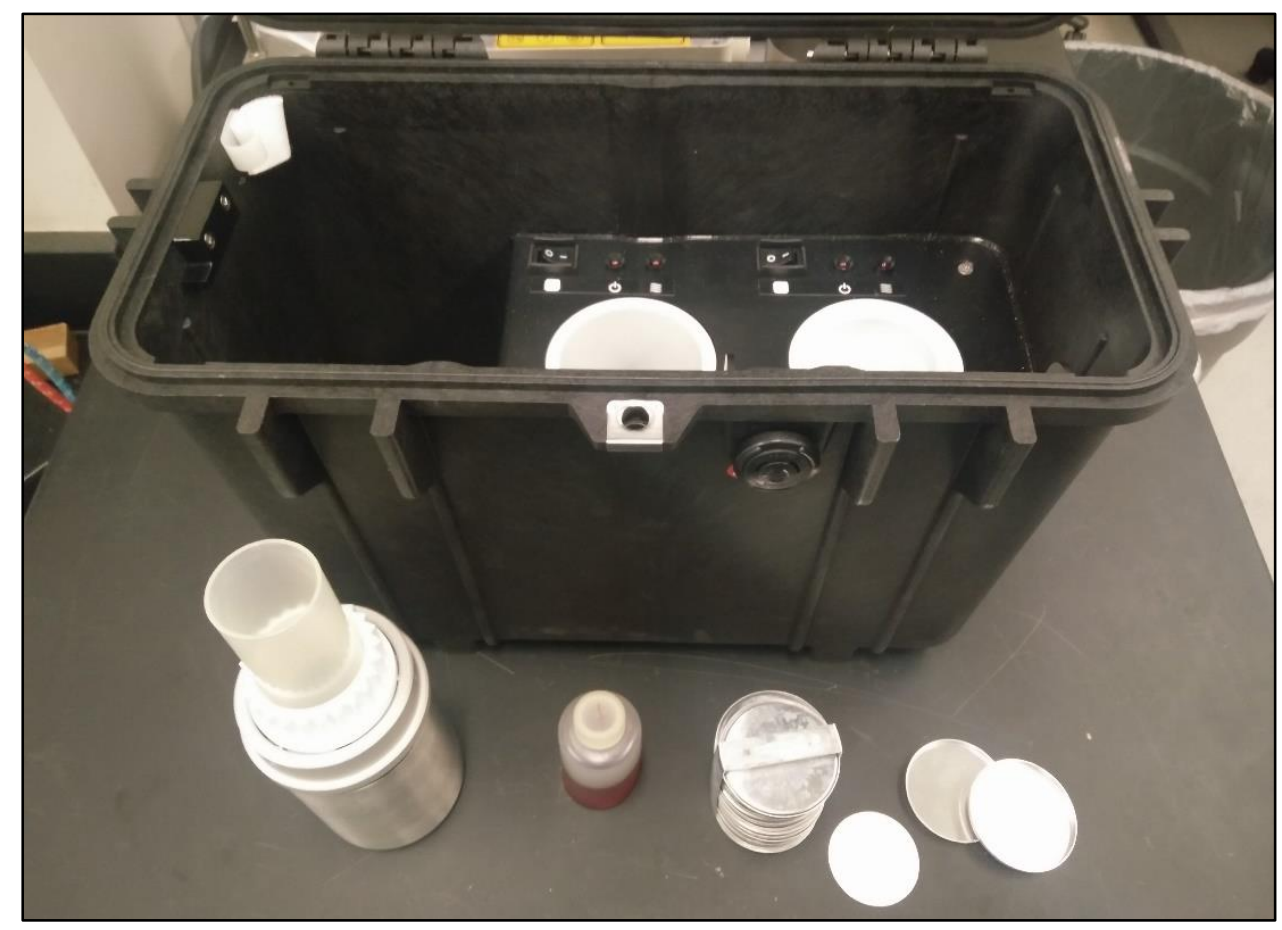

Figure 6: DelAgua water testing kit with Membrane Filtration Vacuum Chamber, MLSB growth medium, aluminum petri dishes, and sterile sample pad

MLSB was prepared using polypropylene bottles provided in the DelAgua water testing kit. The broth was mixed using DI water under laboratory conditions, then sterilized for 30 minutes in a steam sterilizer. The MLSB containers were tightened before the sterilized broth had fully cooled at DelAgua's instruction, which should lead to a 9-week lifetime in cool dry conditions. Bottles of MLSB were not re-used across sampling events, to ensure sterile growth medium conditions at the beginning of each experiment. Following the initial MLSB batch preparation, testing was carried out to ensure similar behavior from two bottles of the solution by sampling one source of river water.

Prior to each sampling event, sterilization was accomplished using a pressure cooker for the aluminum petri incubation dishes and the stainless-steel sampling cup, and 
using a methanol burn for the filtration apparatus and sample cup. The filtration apparatus was also flushed with distilled water prior to each sample. Contamination was controlled for by including a distilled water blank sample between each location sample. In one case, this blank sample was found to be contaminated with TTCs, invalidating that sample for use in the analysis.

Samples were generally taken using $50 \mathrm{~mL}$ or $100 \mathrm{~mL}$ of sample water, depending on recent conditions and previous results from the same site. Two locations with high TTC counts were often taken with $25 \mathrm{~mL}$ sample sizes. At times, the colony count became too high for the results to be considered trustworthy (significantly greater than 100 colonies per membrane), and these results were not included in the analysis. There were also times when multiple colonies appeared to have combined, violating the principle that each colony corresponds to one original TTC cell, and these results were removed from the analysis as well.

In order to control for site variation and prevent sample contamination, the petri dishes were stacked in this repeating order: DI - Stack Blank - 2 Samples. A minimum of 2 samples was tested for each testing location on each testing date.

Samples were incubated at $44^{\circ} \mathrm{C}$ for 18 hours following a 30-minute "rest period" between filtering and incubation, and were counted manually in the case of low coliform counts or through the use of computer-aided visual counting for high coliform counts. Samples were averaged from each location on each date, and standard deviations monitored to ensure that samples were well-mixed and uncontaminated. 


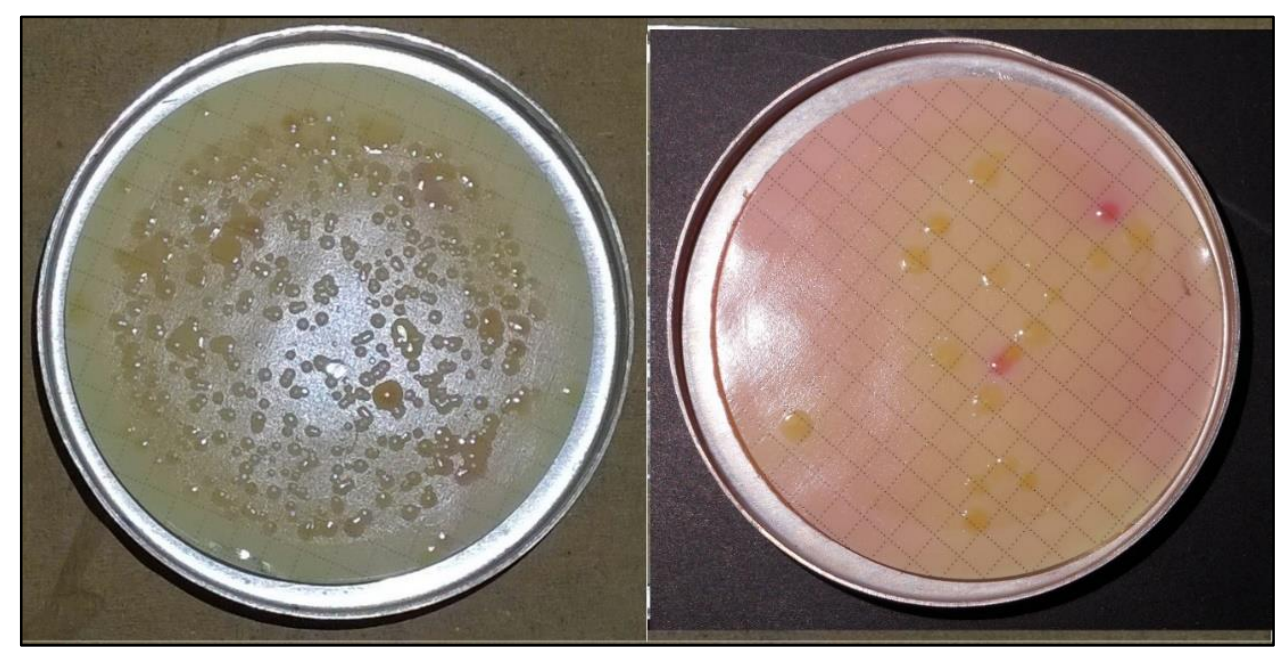

Figure 7: Photographs of Membrane Filtration petri dishes with high colony count and some combined colonies (left) and low colony count (right). Red colonies are bacteria that cannot ferment lactose, and should not be included in TTC counts

In all cases, colonies were counted immediately following their removal from the incubator, as color changes can occur in TTC colonies given sudden changes in ambient temperature. All yellow colonies with a diameter of 1-3mm were considered viable TTC colonies, and were assumed to have resulted from the differentiation of a single cell present in the sample water.

Testing procedures are recounted in more complete detail in Appendix A.

\subsection{SMF4 Fluorimeter Procedure}

The Safe Training Systems SMF4 is a single-excitation, single-emission fluorescence spectrometer designed for field use. The fluorimeter uses a standard $10 \mathrm{~mm}$ sample cuvette, which is inserted into a machined delrin ${ }^{\circledR}$ enclosure and capped with a light shield before triggering a reading. The SMF4 has an emission wavelength set at $280 \mathrm{~nm}$, an excitation wavelength at $360 \mathrm{~nm}$, and a slit width of $10 \mathrm{~nm}(+/-4 \mathrm{~nm})$, corresponding to Peak T2 tryptophan fluorescence. The excitation wavelength is 
generated with a UV LED, and the emission wavelength passes through an optical bandpass filter before being detected by a photomultiplier (PMT). The instrument's output has been verified against tabletop spectrometers in previous literature (Cumberland et al., 2012).

Immediately following each membrane filtration pad preparation, water from the same sample was tested using the SMF4. All samples were taken independently, using sterilized disposable pipettes. The sample bottle was inverted twice between each reading to mix the sample.

SMF4 samples were taken using the "HIGH" sensitivity setting, without tryptophan standard calibration, and are therefore presented in arbitrary units in this analysis. Before the experiment was undertaken, dilutions of fish pond water, river water, and MLSB were tested to ensure a linear response independent of the sample's turbidity, coloring, etc. Samples are taken with a single button press, triggering an intrinsic fluorescence reading in which the excitation LED is turned on and stabilized, then the photomultiplier is used to sample for the fluorescent wavelength. The SMF4 is shown in Figure 8. 


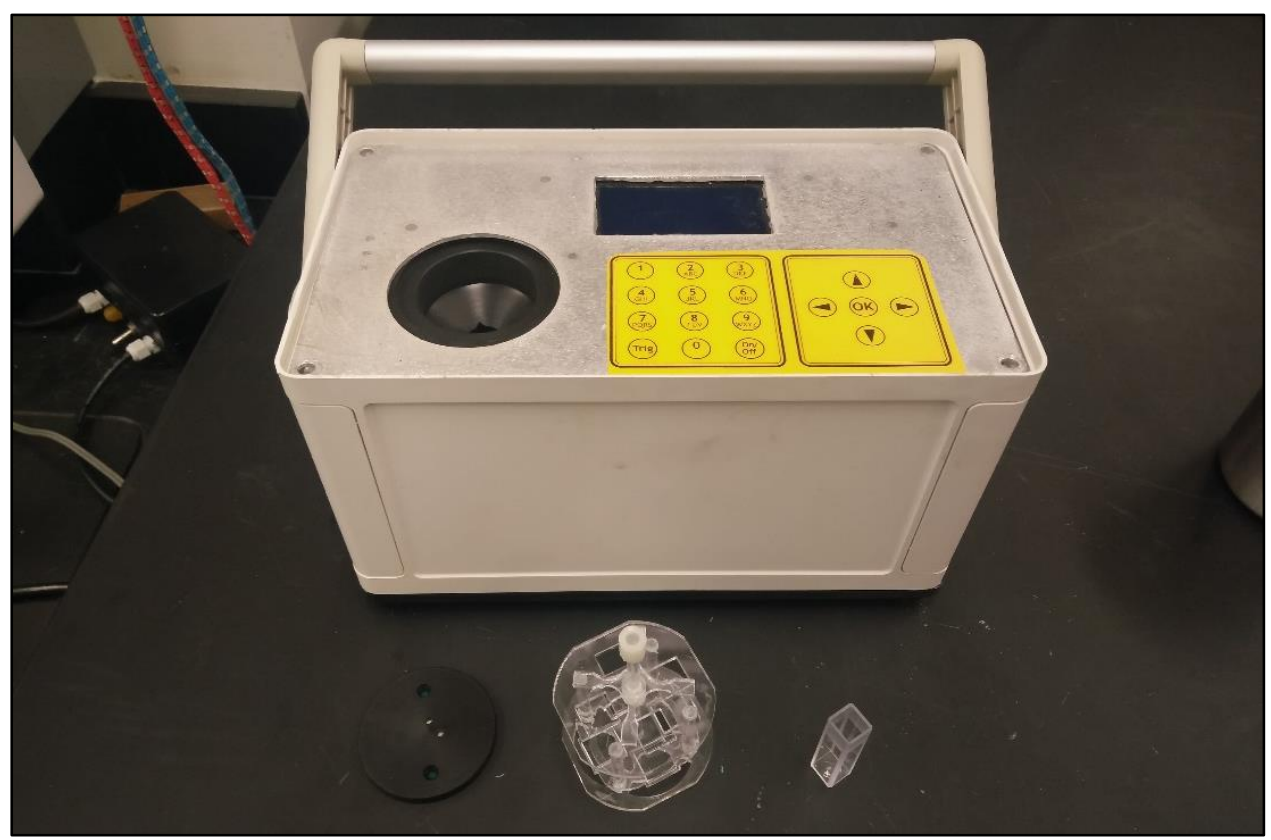

Figure 8: SMF4 Fluorescent Spectrometer with Cuvette, Light Cover, and Custom Cuvette Holder/Cleaner

A UV quartz cuvette (Firefly Scientific) was used for all samples. Between each sample, the cuvette was cleaned using a custom laser-cut cuvette holder submersed and spun in a stainless-steel cup filled with diluted Helmenex III $^{\mathrm{TM}}$ cuvette cleaning solution, cleaned with cotton swabs, rinsed three times with distilled water, and dried with filtered compressed air. The holder prevented the cuvette from cracking and allowed the cleaning to be carried out quickly by turning the cuvette inside the solution and holding it in place during drying. A distilled water sample was then recorded, to be back-subtracted from the sample value and to ensure that the cleaning process had been successful. In total, 4 readings were taken for each sample location on each sampling day. These readings were averaged after back-subtracting the distilled water reading immediately preceding the sample. 


\subsection{Results}

Following training regarding the preparation of MLSB broth, the first batch was tested using the same samples of river water. Six samples plus a DI blank were tested from the same source. The results, shown in Figure 9 below, show similar behavior for all samples.

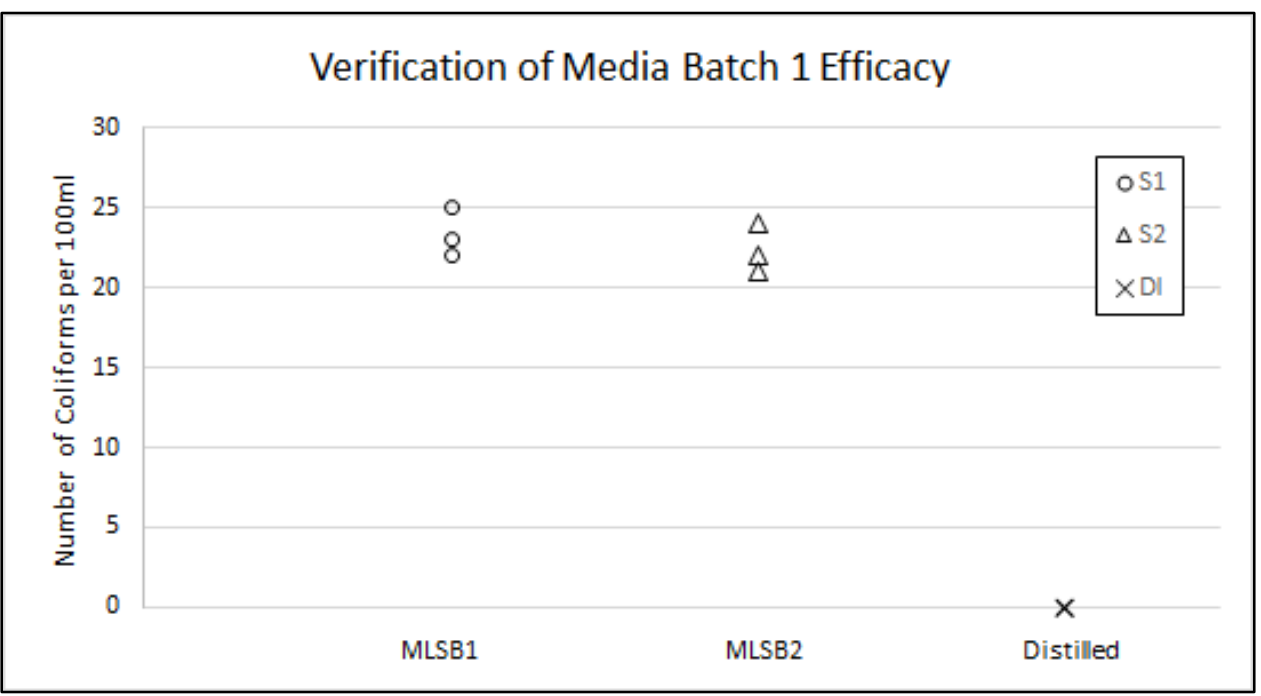

Figure 9: Two media bottles, S1 and S2, were tested together in the dual-incubation chamber using the same water samples to ensure similar results.

Similarly, river water and fish pond water diluted with tap water was tested on the SMF4 to ensure that the output of the fluorimeter changed linearly with tryptophan concentration, rather than with turbidity or color, before sampling was begun in earnest. The results were highly linear in both cases, with the results shown in Figure 10. 


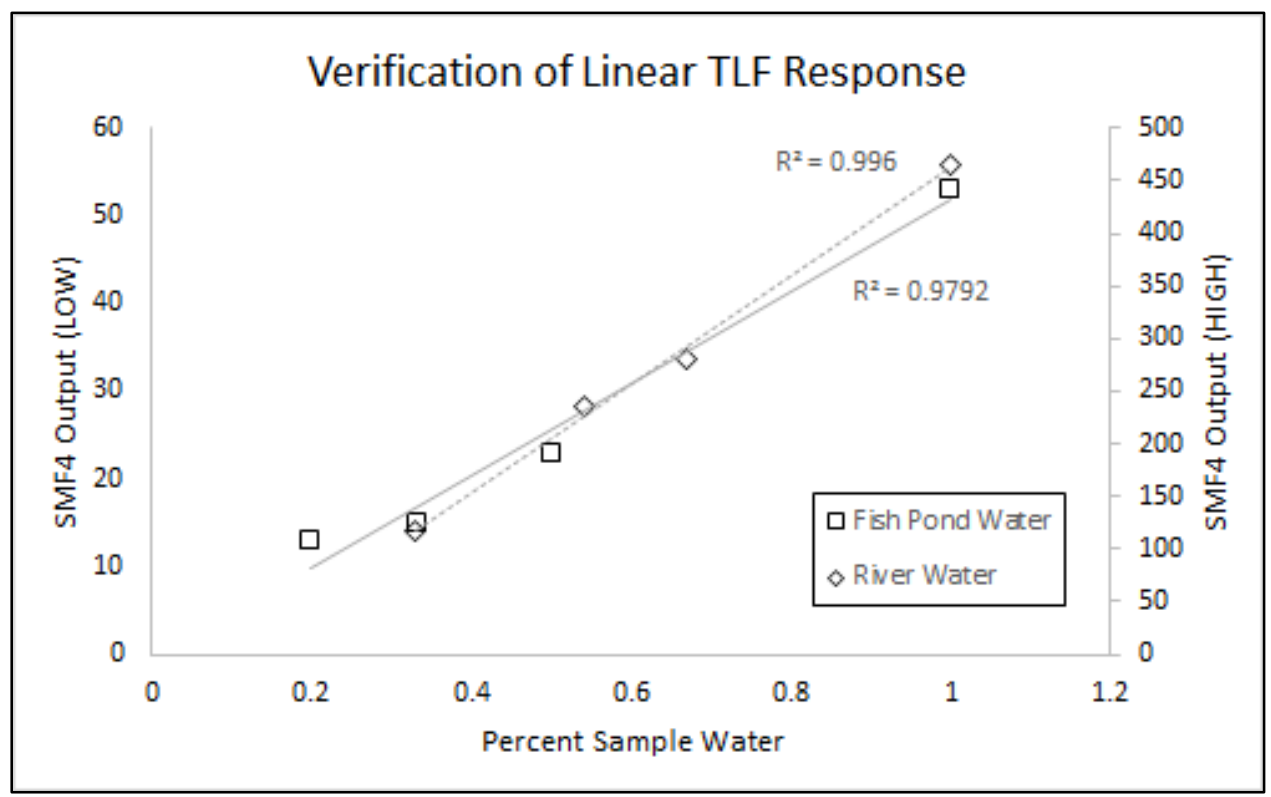

Figure 10: Two water sample sources were diluted and tryptophan fluorescence readings were taken to ensure linear response from SMF4. Values on the x axis are percentage concentrations of sample water to tap water, ranging from $0 \%$ to $1.2 \%$ concentration sample water.

The results of membrane filtration colony enumeration and SMF4 tryptophan fluorescence readings were tabulated and plotted on a log-log scale to assess their strength of association. The results in Figure 11, including a breakdown of sample locations by marker shape and color. 


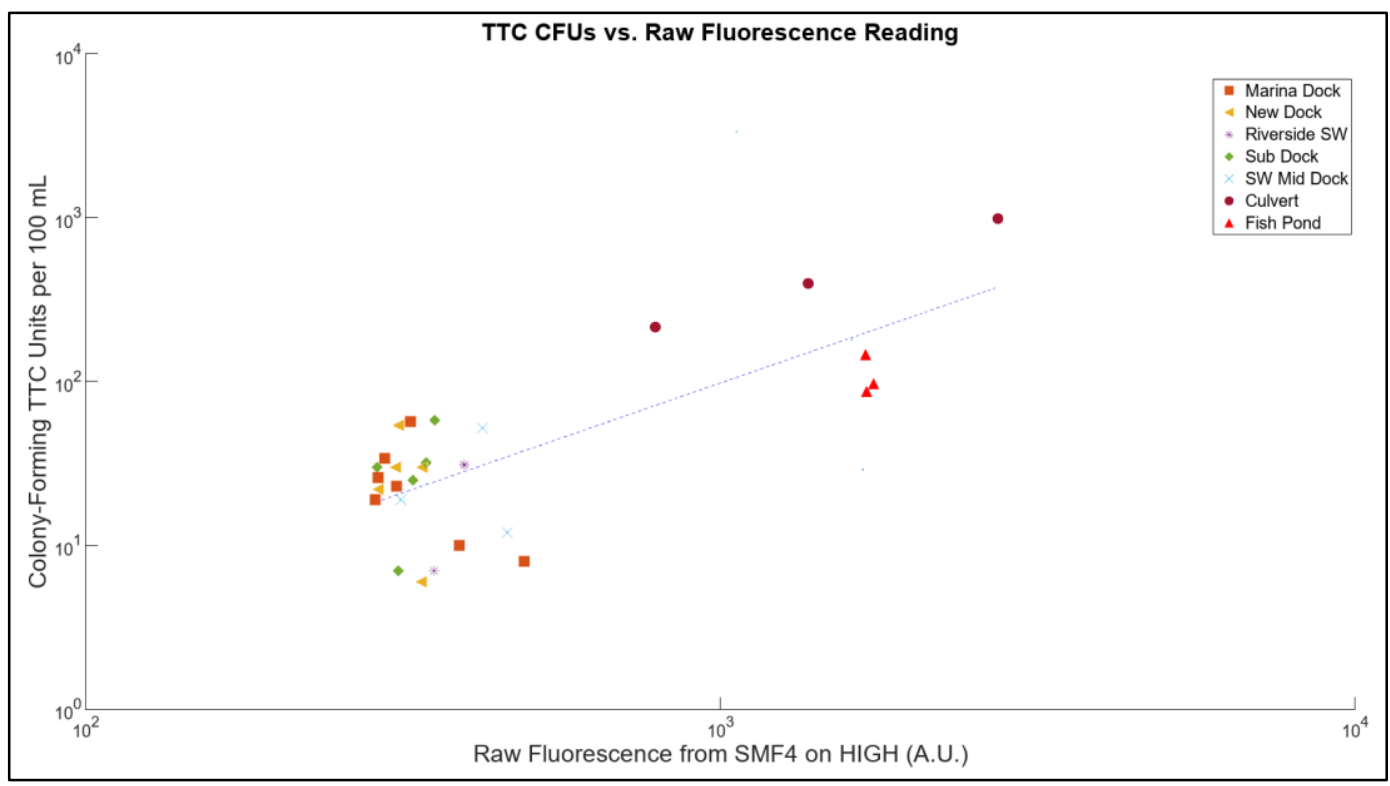

Figure 11: TTC Colony Forming Unit Counts vs. SMF4 Tryptophan Fluorescence Readings. Overall linear correlation is $R^{2}=0.56$

\subsection{Discussion and Comparison to the Literature}

The research conducted here is composed of a significantly smaller dataset than a number of other studies examining the same association. The results of other research, usually reported as $\mathrm{R}^{2}$ values of $\log _{10}$ (fluorescence) and $\log _{10}$ (colony count) or unadjusted colony count, are shown in Table 4 below and compared to the correlation observed in the data collected here. Note that this table does not include the literature which examines the relationships between TLF and other water quality parameters that can be associated with microbial contamination, only those that compare TLF with direct measures of microbial presence or wastewater concentration. 
Table 4: Strength of Association between Microbial Contamination and TLF

\begin{tabular}{|c|c|c|c|c|c|c|c|c|}
\hline & \begin{tabular}{|c|} 
Stedmon et. Al \\
2011 \\
\end{tabular} & $\begin{array}{c}\text { Cumberland } \\
\text { et. Al } 2012 \\
\end{array}$ & $\begin{array}{c}\text { Sorenson et. } \\
\text { Al, } 2015\end{array}$ & \multicolumn{2}{|c|}{ Bridgeman et. Al, 2015} & $\begin{array}{l}\text { Baker et. } \\
\text { Al, } 2015\end{array}$ & $\begin{array}{l}\text { Sorenson et. } \\
\text { Al, } 2016\end{array}$ & $\begin{array}{c}\text { Sharpe, } \\
2017\end{array}$ \\
\hline $\begin{array}{r}\text { Sample } \\
\text { Source }\end{array}$ & Laboratory & $\begin{array}{c}\text { River Water } \\
\text { \& Sewage } \\
\text { Effluent }\end{array}$ & $\begin{array}{c}\text { Groundwater } \\
\text { (potable) }\end{array}$ & \multicolumn{2}{|c|}{ Various (potable) } & $\begin{array}{c}\text { River } \\
\text { catchments }\end{array}$ & $\begin{array}{c}\text { Groundwater } \\
\text { (potable) }\end{array}$ & $\begin{array}{l}\text { Surface } \\
\text { Waters }\end{array}$ \\
\hline Analyte & $\begin{array}{c}\text { Wastewater } \\
\text { Concentration }\end{array}$ & E. coli & TTCs & E. coli & $\begin{array}{l}\text { Rhodococcus } \\
\text { species }\end{array}$ & E. coli & TTCs & TTCs \\
\hline $\begin{array}{r}\text { Microbial } \\
\text { Test }\end{array}$ & $\mathrm{n} / \mathrm{a}$ & Colilert 18 & $\begin{array}{l}\text { Membrane } \\
\text { Filtration }\end{array}$ & $\begin{array}{l}\text { Membrane } \\
\text { Filtration }\end{array}$ & $\begin{array}{c}\text { Flow } \\
\text { Cytometry }\end{array}$ & $\begin{array}{l}\text { Colilert } \\
\text { (IDEXX) }\end{array}$ & $\begin{array}{l}\text { Membrane } \\
\text { Filtration }\end{array}$ & $\begin{array}{c}\text { Membrane } \\
\text { Filtration }\end{array}$ \\
\hline $\begin{array}{r}\text { Number of } \\
\text { Samples }\end{array}$ & 7 & 25 & 117 & 190 & 48 & 128 & 150 & 28 \\
\hline Result & $R^{2}=0.97$ & $R^{2}=0.72$ & $R^{2}=0.57$ & $R^{2}=0.11$ & $R^{2}=0.86$ & $r=0.74$ & $\rho=0.554$ & $R^{2}=0.56$ \\
\hline
\end{tabular}

It is important to note that the relatively strong association shown in the data in this analysis is not reflected on a single-site basis. This may be the result of the limited dataset for each individual sampling site. A meaningful study in the future would be to compare these results to a larger dataset collected all at one source of groundwater over a similar time period. It is possible that the variance in both properties for one given location is low enough that a much larger dataset is indeed necessary to identify significant changes in these properties.

One serious issue encountered in this analysis is the inability to overlay the data generated in this analysis with the data generated from previous explorations of the association between TLF and TTC abundance. If a common standard is not established immediately prior to testing, using tryptophan standards, the results will be reported in arbitrary units, as is the case in this thesis. It is therefore important, as this work continues, for researchers to calibrate their fluorescent spectrometers to tryptophan standards in order to avoid the issues presented by degraded photomultipliers, photodiodes and light sources. 


\section{Section 4: Prototype Development}

The most promising application of a tryptophan fluorescent sensor in the context of drinking water monitoring is as a member of an array of on-line, in-situ sensors capable of detecting early warning events in drinking water distribution systems. This is due mainly to the short time required to complete a test and quantify the tryptophan concentration in a sample.

A second promising application is the use of such sensors in rural water distribution systems, which are often comprised of a single hand pump or a larger, electric-pump borehole. Sampling large groups of rural water points is expensive and difficult to manage, often incurring significant costs in terms of transportation alone. In addition, sample transportation to a suitable location for analysis under sterile conditions imposes real logistics challenges. One option is to replace spot testing with on-line monitoring sensors, a concept made even more appealing by the ability for fluorescence sensors to "smooth out" seasonal variations in microbial water quality (Sorenson et Al, 2015).

In both of these use cases, a tryptophan spectrometer that can quickly interface with existing water distribution piping systems is called for.

\subsection{Target Capabilities}

Given the literature's consistent finding that TLF is associated with microbial contamination, the prototype should be capable of detecting changes in tryptophan concentration as a proxy for the presence of microbes. Initial sensitivity goals could be based on the minimum changes in tryptophan concentration associated with changes in 
microbial contamination level in (Baker et al., 2015). Specifically, the sensor should be able to maintain a linear relationship between output signal and tryptophan content down to the level of $1 \mathrm{ppb}$ tryptophan, with the ability to differentiate between levels of $0,1,10$, 100 and $1000 \mathrm{ppb}$. This is based partially on the goal of attaining a sensitivity between 0 and $3 \mathrm{ppb}$ tryptophan, the cutoff point chosen to accurately predict high levels of contamination (>100 CFU per $100 \mathrm{~mL}$ ) with 94\% accuracy. Further research will be necessary to determine whether this sensitivity is sufficient to detect contamination events.

Other design considerations include mounting systems to accommodate a variety of water resource transportation systems; enclosure development for the protection of the electronics; and form factors which minimize both device profile and cost. An ideal sensor should be able to consistently sample a water source for an extended period of time without the need for hands-on maintenance; should be capable of triggering an alarm if a pre-set threshold of tryptophan fluorescence is exceeded; the alarm should be transmitted wirelessly to an alert system that can provide information about a contamination event within a narrow window of time; and the system should be reproducible for a low cost in order to make a large-scale, networked solution tenable in both municipal and remote environments.

Since tryptophan fluorescence can be associated with high levels of bacterial activity, it is a strong candidate for a sensing mechanism that could detect contamination events and trigger an early-warning system. However, since most water distribution systems are comprised of hundreds to thousands of nodes, tracking contamination events 
back to their source is a daunting task. Fortunately, researchers have also been active in this management task, in anticipation of the development of an on-line water quality sensor. For example, Xu et Al. (2008) have developed a methodology for the placement of a small number of sensors to monitor a much larger network. In their study, simulations based on this methodology indicated a $77 \%$ detection rate for the placement of 10 sensors in a network of 129 total nodes. Detection rates quickly increased as sensor placements increased, offering strong evidence that even complicated distribution systems could be monitored with high levels of success given the development of an effective early-warning sensor technology.

\subsection{Design Difficulty: Biofouling}

A major issue for any in-situ optical sensor is the accumulation of microbial biofilm over the sensing window. The buildup of this material can have effects which are difficult to predict for a fluorescent sensor, since the biofilm may fluoresce at the same wavelengths as tryptophan, increasing the signal, or may attenuate the signal. Traditional mechanical components including wiper blades and copper shutters are unattractive for long-term in-situ designs. Novel optical window materials which prevent biofilm adhesion may prove beneficial to the technology; another solution might be the application of algorithms which automatically correct for fouling during in-situ deployment.

\subsection{Design Difficulty: Component Costs}

Most fluorescent spectrometers make use of a series of bandpass filters to select excitation and emission wavelengths, using a xenon or halogen lamp as an initial light 
source. In addition to high power consumption, the series of optical components required for the emission portion of the device incurs significant costs. In the past 10 years, deepUV LEDs have become commercially available, with lower power consumption, low levels of degradation, and very tight emission bands (often centered around one wavelength $+/-5 \mathrm{~nm})$.

Similarly, expensive photomultipliers traditionally used for the detection portion of the device are now at times being replaced by much lower-cost photodiodes, semiconductor devices which generate a current based on incident light. Semiconductor technology can significantly drive down the cost of both emission and detection sections of a spectrometer, although this comes at the cost of a significant loss in sensitivity. As UV LEDs continue to increase in power output, this difficulty can also be expected to be overcome in steps.

\subsection{Sensitivity Requirements and Factors Affecting Sensitivity}

Current research using log-linear correlations between colony counts and tryptophan fluorescence shows sensitivities in the range of $10-100 \mathrm{CFU} / 100 \mathrm{~mL}$, which is not within WHO's guidelines for drinking water quality determination. Therefore, this technique may be more suitable when sampling occurs at regular short intervals - not as an individual cell counting mechanism, but rather to sense sudden changes in tryptophan

fluorescence concentration, which may be a proxy for a contamination event. Sorenson et al. (2015) found that in presence-absence tests, median TLF concentrations were 0.9 and $9.2 \mu \mathrm{g} / \mathrm{L}$ based on tryptophan standards - a sensitivity requirement well within the capabilities of both field and laboratory fluorescent spectrometers. 
However, sensitivity of the instrument is affected by both temperature changes and turbidity as explored by Khamis et al. (2015), as well as by chemical characteristics of the sample, including dissolved oxygen content. Some of these issues may be minimized by the expectation that drinking water is of significantly lower turbidity than surface water examined in the literature, but thorough testing will be required to identify the severity of this effect, along with the stationarity of groundwater temperatures.

\subsection{Prototype I Design}

Like most portable products, the SMF4 is a cuvette-based field fluorimeter, not suited for in-line sensing. The components of the SMF4 were repackaged into a 2-inch flow-through design which can be spliced directly into a water distribution line.

A drawing of the prototype and its components is shown in Figure 12. A sampling chamber protrudes from the lower side of the apparatus, and the photomultiplier and UV LED are perpendicular to one another. This sampling scheme was chosen in order to find a solution that would work for either filled or unfilled pipes, and in order to increase the accessibility of the sampling chamber components. A single optical bandpass filter serves as one window along the axis of the PMT, with a polished silica optical window along the axis of the light source. A PMT of the same model is used as a detector, while the UV LED from the SMF4 is used as the emitter. Components provide pressure against sealing o rings. The body of the prototype is comprised of UV-stabilized PVC, with a machined enclosure that seals against a standard 2-inch PVC threaded pipe with pressure from stainless-steel laser-cut mounting plates. 


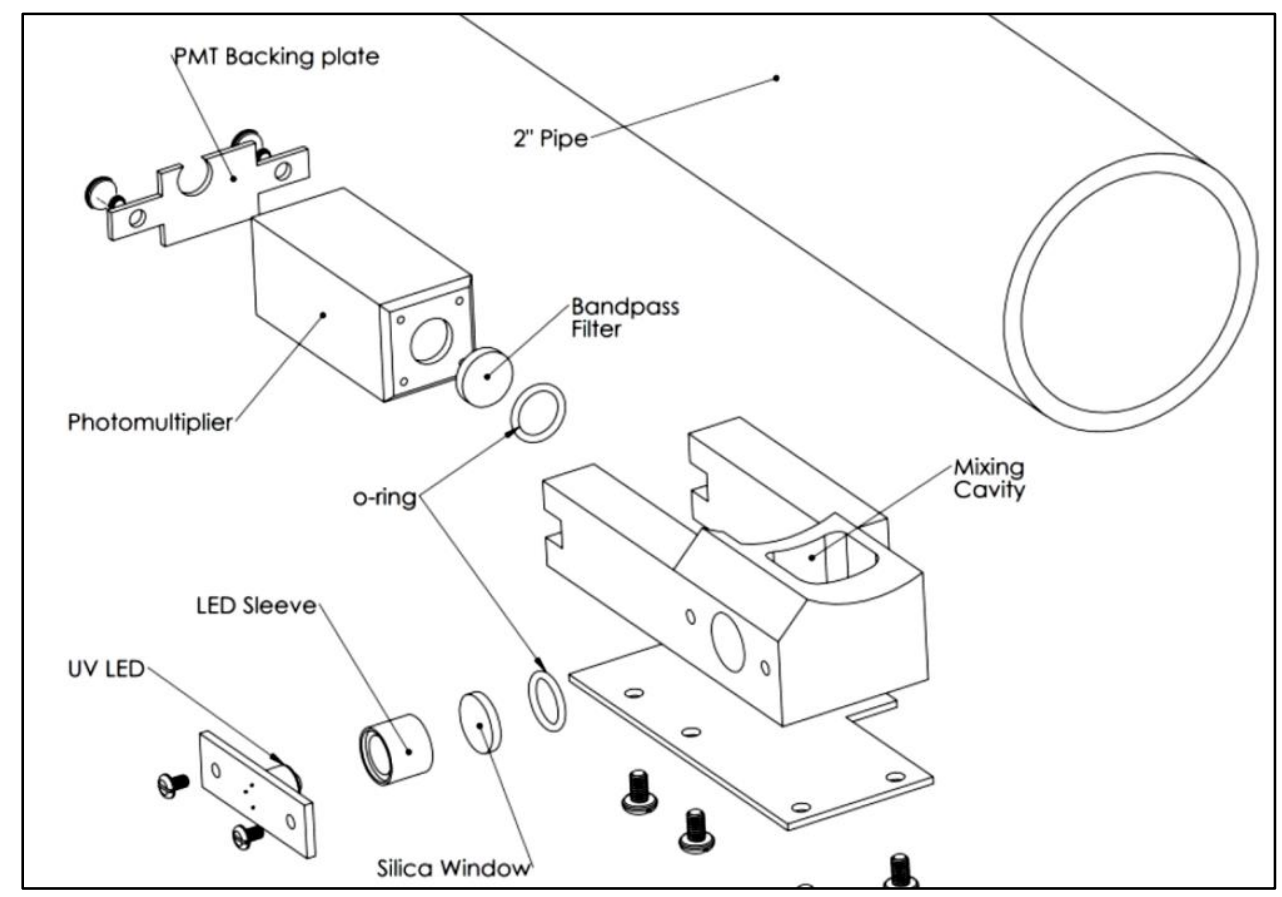

Figure 12: Exploded view of Repackaged Components from SMF-4

Design requirements for the prototype were that the prototype should work in filled or partially-filled water lines, or a standard size (2"); that the optical components should be easily accessible, and maintain compatibility with the geometries of the SMF4's photomultiplier and circuit-board mounted UV LED; that the sampling chamber would remain watertight under static and flow conditions; that water in the sampling chamber should experience low residence times (below 1 minute); and that the impact on the overall flow of the water line would be minimally disruptive.

\subsection{Prototype I Residence Time Calculations}

Of particular interest in a flow-through, high-frequency residential water quality sensor is the rate at which water leaves the sampling chamber and is replaced by water from the source side. An analysis was completed with the simplifying assumption that the flow in the tube could be approximated as a flow over a rectangular cavity with no radii. 
Two literature sources were found to explore this scenario, and their results are compared below. The models are analogous to the constraints of the prototype design, but differ in some ways, as shown in Figure 13 and Figure 14. The most significant differences from a fluid dynamics perspective are the flat floor profiles above the mixing cavity, and the lack of machined radius corners in the numerical simulations.

The models are used to predict ejection time from the cavity, primary hydraulic residence time, and secondary hydraulic residence time (for an initially-stationary particle in the chamber). Both models rely on dimensionless parameters including the Reynold's number and ratios of length to depth and height to depth of the cavity and the stream. Note that both models are for the case of a flat fluid stream, and do not take into account either the radius of the 2 " pipe or the radii present in the milled sampling chamber. The question at hand is whether the time spent in the cavity is below 1 minute in order to allow a temporal resolution of 1/60 hertz.

In the literature, this problem is referred to as "driven cavity flow". The central question is how the flow of liquid above a square cavity affects the mass transport of fluid within the cavity itself. In general, the approach is to model the vortex formations that are expected to occur within the cavity, and to establish numerical and experimental models for the residence time of particles within the cavity. The literature generally agrees that in the case of significant depth of the cavity, one or two main vortexes and two secondary ones can be expected to form.

Chang et al. (2006) used numerical simulation using an advective-diffusion equation to examine the effects of flow over a scalar particle set in a cavity of height D 
with length 2D. The simulation used the incompressible form of the Navier-Stokes equation, and found two main vortexes in the case of both laminar and turbulent flow conditions. The simulation was used to explore the effects of dimensionless parameters and velocities on ejection of initially-stable particles from the cavity. The simulation domain is shown in Figure 13.

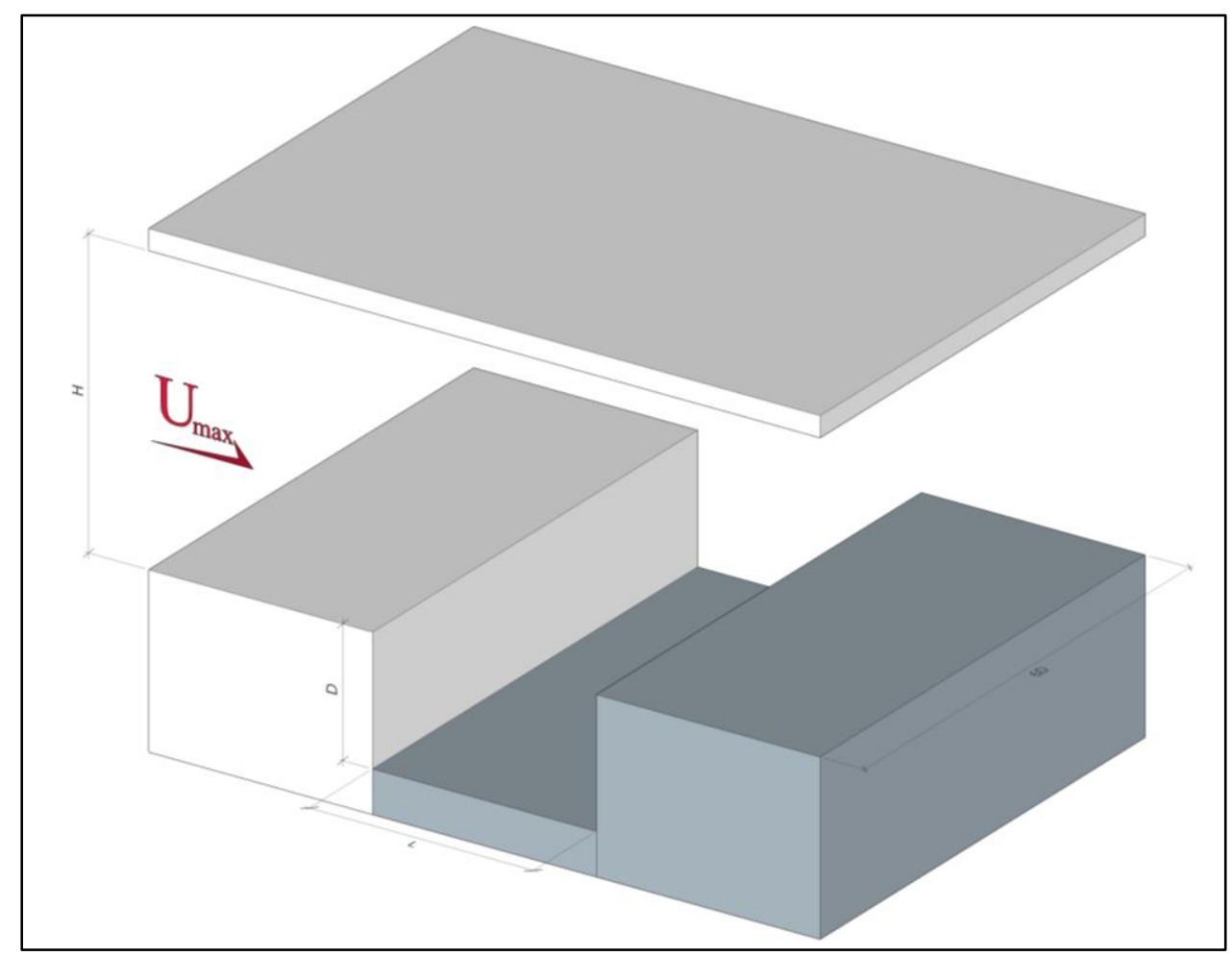

Figure 13: Simulated Geometries for Chang et al. (2006)

The model assumes a value of $\mathrm{L} / \mathrm{D}=2, \mathrm{H} / \mathrm{D}=6.01$, and the overall Reynolds Number with respect to the cavity, $\operatorname{Re}_{\text {D.Cavity }}=3360$. In order to generate an estimate of the ejection time for the case of $\mathrm{L} / \mathrm{D}=2$, the Reynold's number for the cavity was adjusted. In the case of the model, we have, $\frac{L}{D}=2, \frac{H}{D}=6.01$, and $R e_{D \text {.cavity }}=3360$. In the case 
of Prototype I, we have, $\frac{L}{D}=1, \frac{H}{D}=2.86$, and $R e_{D \text {.cavity }}=1622$.

Substituting these values into the model and solving for the time required for $90 \%$ ejection of scalar from the cavity, we have,

$$
t_{90 \%, \text { turbulent }}=\frac{166 D}{U_{\min }}=26.4 \mathrm{~s} \text { and } t_{90 \%, \text { laminar }}=\frac{208 \mathrm{D}}{U_{\min }}=33.1 \mathrm{~s}
$$

Drost et al. (2014) examined the mean residence times in rectangular cavities, in the context of natural streams. Their model uses the 3-dimensional Navier-Stokes equation, and their results are validated using experimental data for a variety of width-tolength ratios and a variety of Reynolds numbers. The simulation geometry is shown in Figure 14.

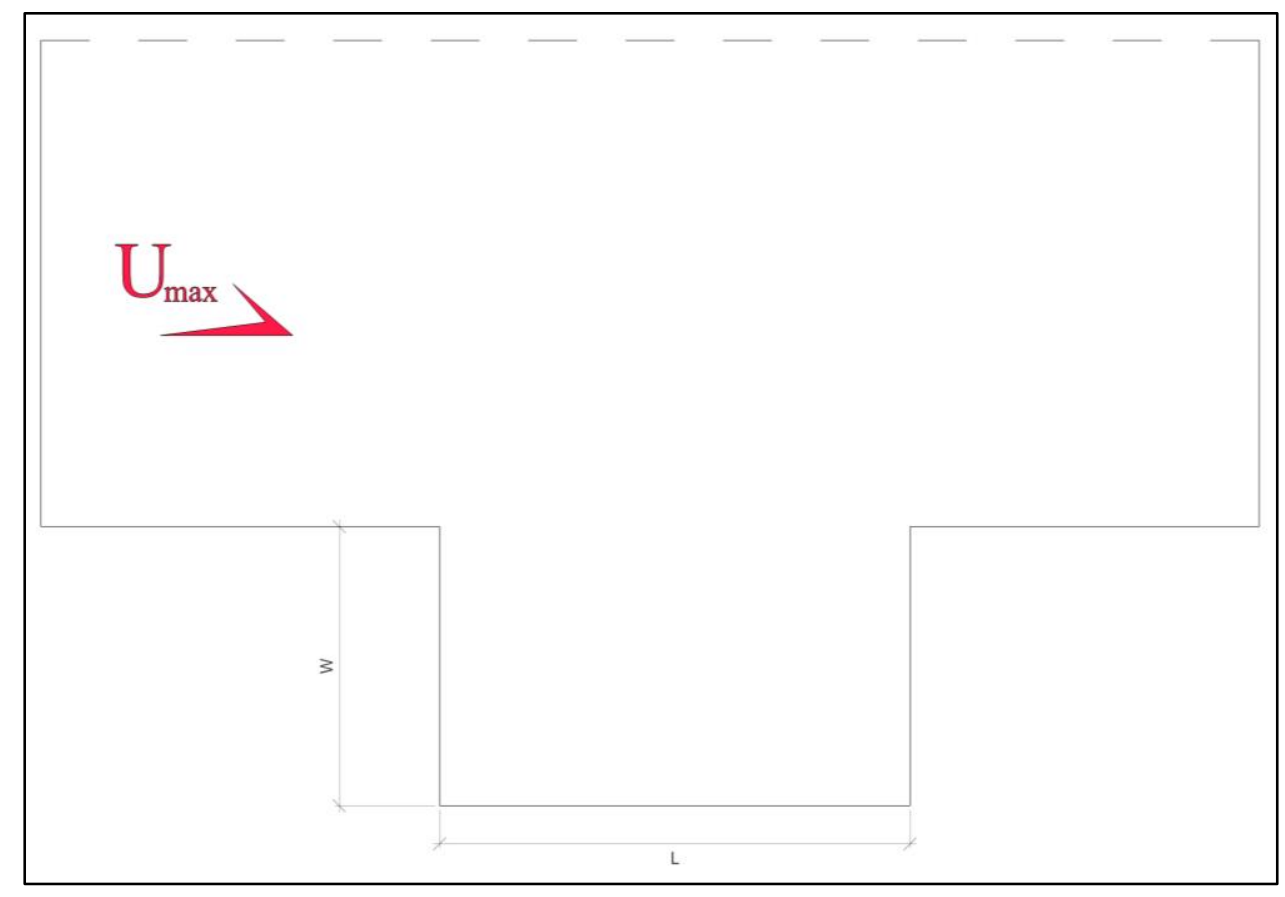

Figure 14: Simulated Geometries for Drost et al. (2014)

Their model is applied to estimate the residence times of particles in the sampling 
chamber of Prototype I.

For prototype 1, we have, $H=0.779 \mathrm{in}, L=0.73 \mathrm{in}, D=0.73 \mathrm{in}, T_{\text {conv }}=\frac{H}{U_{\min }}$. $\mathrm{T}_{\text {conv }}$ is the "convective time scale", or the time in which fluid travels across the length of the cavity, and is related to the simulated residence times in the cavity by,

$$
\tau_{p}=T_{\text {conv }}\left(15 \frac{H^{\frac{3}{4}}}{L}\left(\frac{U_{\min } D}{v_{\text {water }}}\right)^{\frac{1}{4}}\right)=18.9 \mathrm{~s} \quad \text { and } \quad \tau_{s}=T_{\text {conv }}\left(170 \frac{H^{\frac{3}{2}}}{L}\left(\frac{H}{D}\right)^{\frac{1}{2}}\right)=35.8 \mathrm{~s}
$$

where $\tau_{p}$ and $\tau_{s}$ are the primary and secondary residence times within the chamber, representing two perfectly mixed CSTR regions - one in the perimeter of the main eddy, and the other in the core of the vortex region.

In both cases, these estimates indicate that the target time resolution of the cavity as designed should fall within the one-minute sampling window desired.

\subsection{Prototype I Optical Components Testing}

The response of the SMF-4 and the repackaged sensor design was compared using MLSB dilutions of known concentrations. MLSB dilutions were taken in succession from the same bottle, transferring the UV LED from the SMF4 to the prototype; permanent photomultipliers were used in both prototypes. The photomultiplier used in the prototype was of significantly greater age and had been used over many more cycles, affecting its performance in comparison to the one in the SMF4. The results are shown in Figure 15 . 


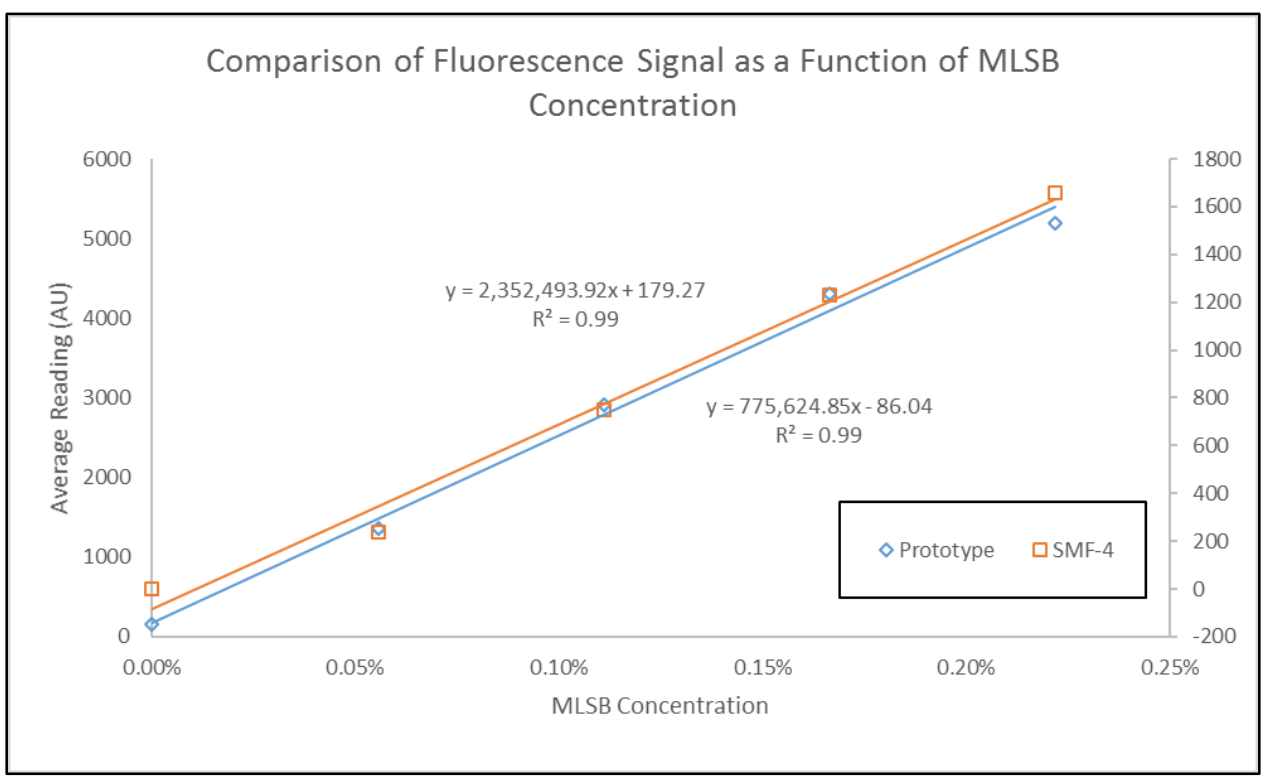

Figure 15: Comparison of linearity of signal response between SMF4 and Prototype 1 sampling chamber

Both cavities show a highly-linear response as MLSB, and therefore tryptophan, content in the sample increases $\left(\mathrm{R}^{2}=0.993 \mathrm{SMF} 4,0.989\right.$ Prototype). This indicates that the concept of using an optical bandpass filter as both a viewing window and a waterproofing element may hold promise for future prototypes, especially in situations where the filter can be cleaned periodically to prevent mineral scaling or biological fouling.

\subsection{Prototype I Issues}

A full analysis of the first prototype was not completed, with no tryptophan standards compared against the results from a lab spectrometer. In addition to this, readings were taken only in a static condition; further tests during flow conditions could be used to experimentally test the results from section 4.6, but would require a faster sampling resolution than the one provided by the SMF4's circuit board and firmware.

No lensing system was included in the design. This, in addition to the use of an 
aging photomultiplier, led to significantly-lower sensitivity than that in the SMF4. It is also likely that the specificity of the system would degrade significantly in in-situ deployment as the bandpass filter's optical coating could degrade over time being directly exposed to water.

Additionally, biological fouling might be accelerated by stagnant water in the sample chamber during long periods of no-flow condition. It is likely that biological fouling's significant and unpredictable impacts on fluorescent signal would make a design like this one useful only for short-term deployments.

\subsection{Prototype II Design}

A second prototype was developed based on a concept intended to help optimize component placement. In short, individual optical components - emitter, converging lens, bandpass filters, and detector - can be individually moved without changing the location of other components. This is accomplished by machining and substituting in new blocks which vary in length or geometry. For example, some fluorimeters are designed to flood as much of the sample as possible with light from the emitter, requiring the emitter to be placed some distance from the cuvette; others minimize the effects of scattering by placing the emitter within millimeters of the cuvette. Figure 16 shows how the second prototype can be tested in both of these configurations by exchanging a short UV LED block for a long one. 


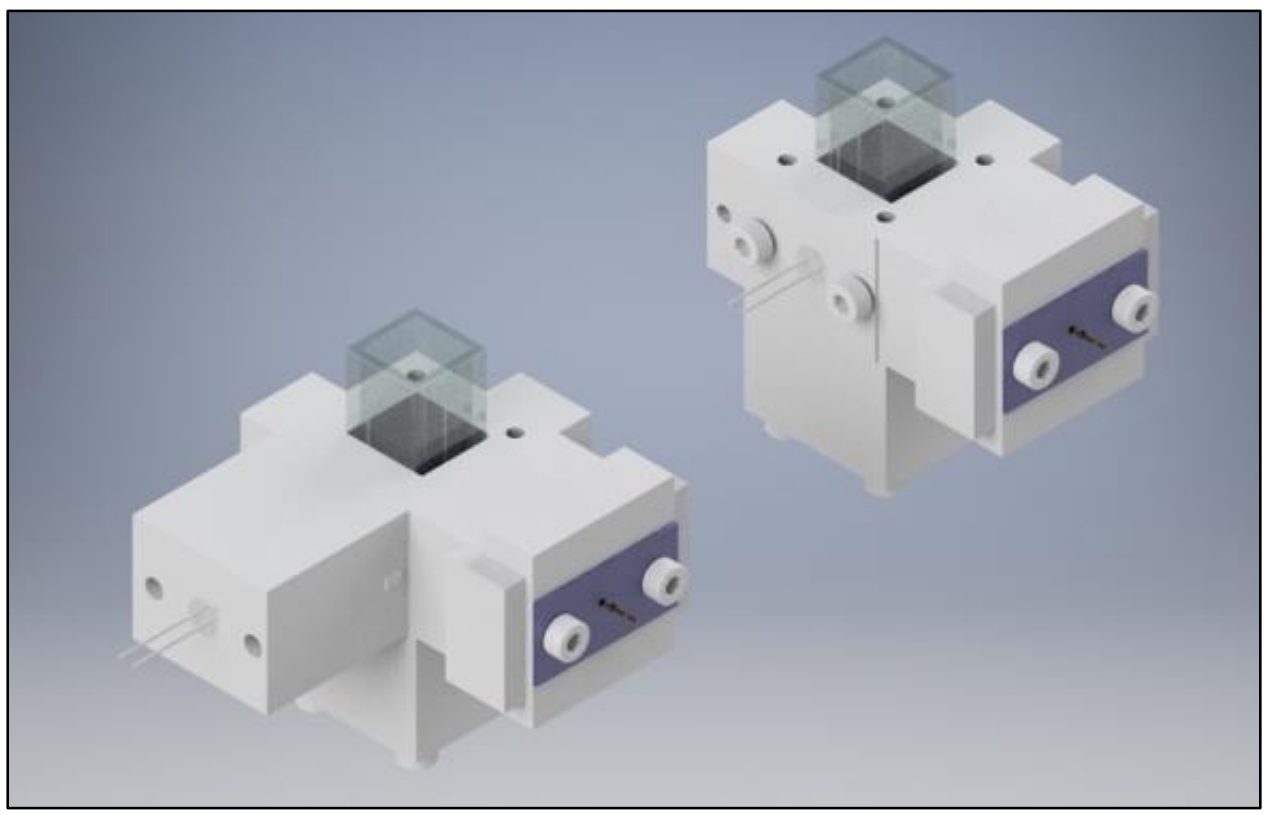

Figure 16: Software Render of Prototype II with a Long UV LED Block (left) and a Short UV LED Block (right). All blocks can be replaced with others of varying geometries and with varying components

Each block is machined from black Delrin ${ }^{\circledR}$, a highly-machinable, self-lubricating acetal resin material. Because each component is housed in a separate block, new blocks can be included or removed to experiment with new optical components or with new geometric arrangements of existing components. The design is such that for many of these blocks, external tolerances are of little importance, allowing new configurations to be machined more quickly if certain constraints are met.

Prior to machining, a 3D printer was used to check the fits of each component. The 3D-printed assembly is shown in Figure 17. The cap seen at the top of the assembly can be removed to swap out cuvettes between sampling events. 


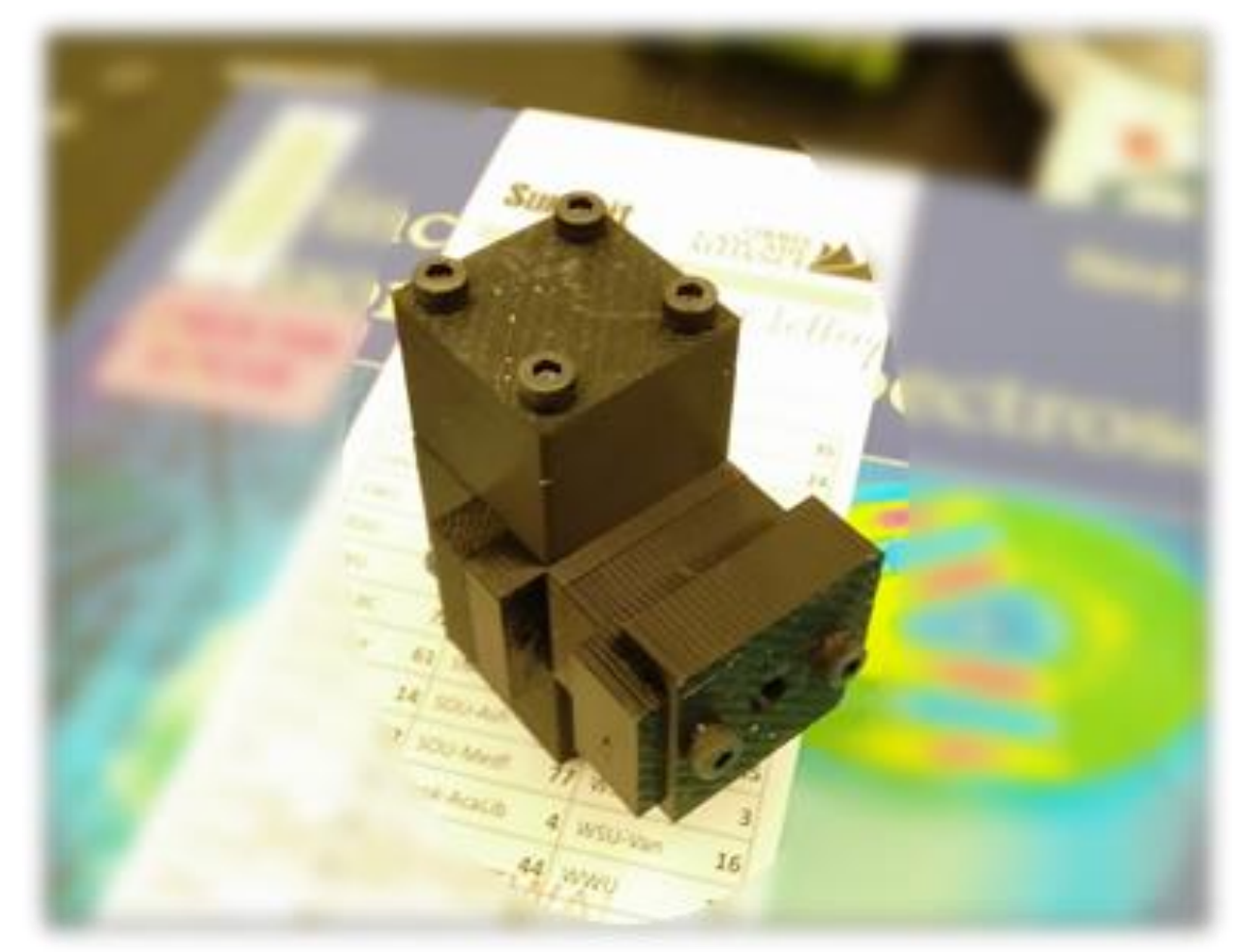

Figure 17: 3D Printed Prototype II Assembly, manufactured prior to machining

A single sampling cuvette is installed in the prototype, and can be removed and replaced by loosening 4 screws and removing a cap. New advances in UV-transparent polymers offer the promise of disposable cuvettes for use at these wavelengths; these sampling chambers could be swapped out on a monthly basis in order to account for biofouling of the chamber. In addition to this, high pressures that might crack the glass of UV silica or quartz sampling chambers could be decreased with the inclusion of a small drilled hole at the end of the polymer cuvette. Another possibility is to use a flowthrough cuvette, open at both ends, with UV-transparent silica or quart surfaces. The interface between the sampling chamber and the water source has not yet been fully designed.

Prototype components include a UV LED centered around 280nm (Marktech 
Optoelectronics) with no secondary optical bandpass filter; a UV Photo Diode centered around 350-360nm (Marktech Optoelectronics), a double converging lens with UV transmission coating (Edmund Optics), and an emission bandpass filter (Edmund Optics). Components are positioned according to their varying focal lengths, and the emission beam is concentrated in a $1 \times 1 \mathrm{~mm}$ detection surface on the photodiode.

The prototype is designed to interface with a saddle clamp, allowing it to be installed on a variety of plastic water pipes, as shown in the drawing in Figure 18. The sensor should be mounted at roughly a 45-degree angle with respect to the horizontal, meaning that the sampling chamber will only fill in the event that the pipe fully fills during flow events; the chamber will then empty again once the flow event has completed. These design constraints vary considerably from the design of the first prototype. 


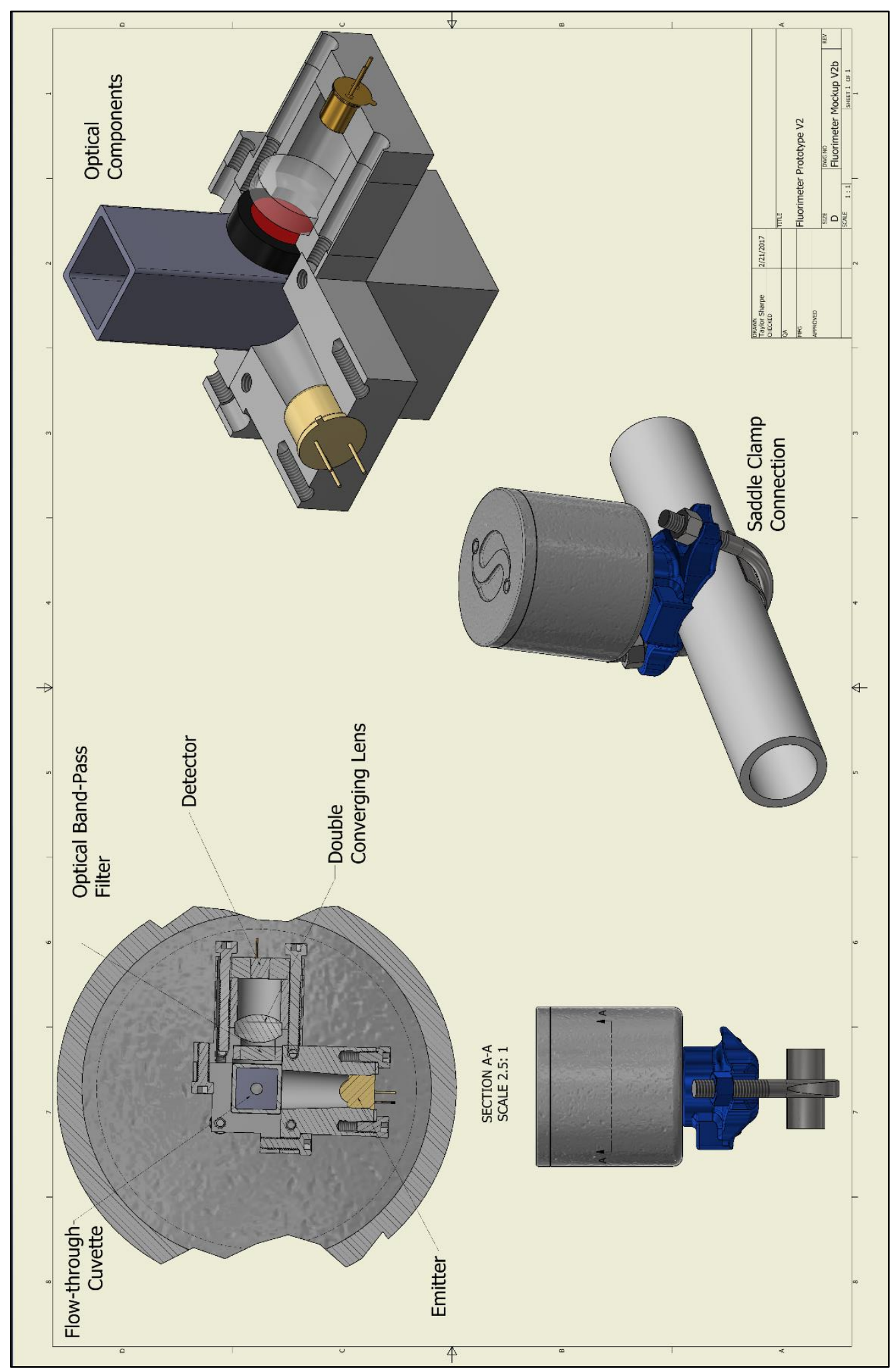

Figure 18: Rendered Drawing of Prototype II 


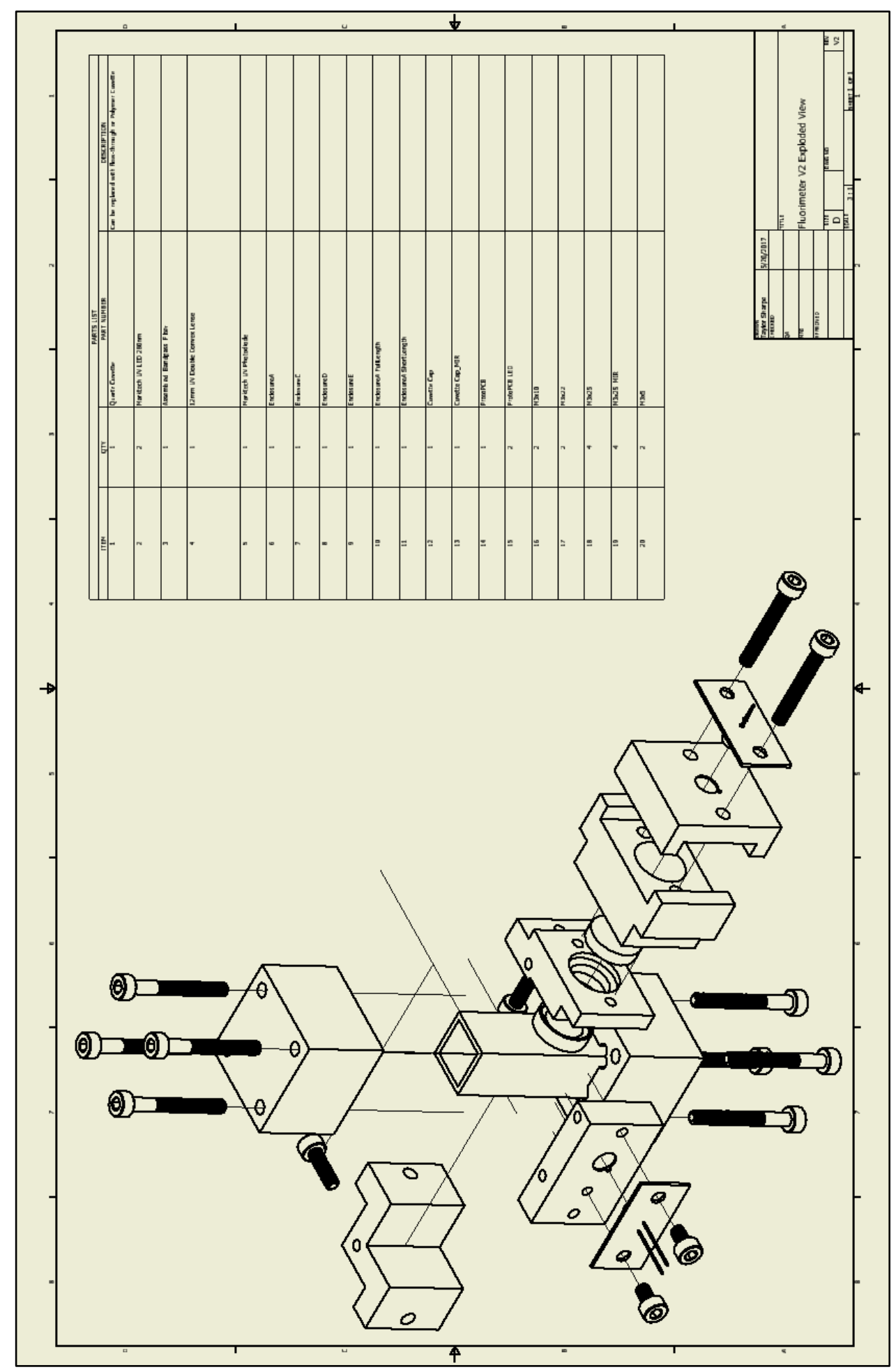

Figure 19: Exploded View of Prototype II, showing top cap, exchangeable enclosures, emitter, detector, and optical equipment 


\subsection{Prototype II Testing}

The sensitivity goals of the prototype are in line with the requirement addressed in 4.1 Target Capabilities. The prototype should ideally be capable of differentiating between $0,1,10,100$, and $1000 \mathrm{ppb}$ tryptophan concentration in distilled water samples.

The prototype went through initial testing in no-flow conditions, in which prepared tryptophan standards were inserted in a standard quartz cuvette. A set of amplification circuits were developed and the testing carried out by D. Wilson and S. Croshere at U.C. Berkeley. The emitter was brought to a constant current state for 5 seconds before a reading was taken with the detector and amplified to be read with a picoammeter (Tektronix, Inc.). The tryptophan standards were diluted from a 100ppm starter made with L-Tryptophane (Acros Organics) and DI water under controlled conditions, mixed with a heated shake plate. Each dilution was mixed in a pre-washed glass vessel and stored in a cool dark place overnight. Dilutions from 0 to $10,000 \mathrm{ppb}$ were prepared in multiples of $10 \mathrm{ppb}$. All testing was carried out the following day.

Samples were taken by following procedures similar to those developed in earlier parts of this research: the cuvette was thoroughly cleaned using Helmenex III and flushed multiple times with DI water before dilutions were pipetted into it and readings were taken using the prototype. No back-subtraction was performed during this series of experiments. A total of 6 tryptophan standards are included in this analysis. The results are shown in Figure 20. 


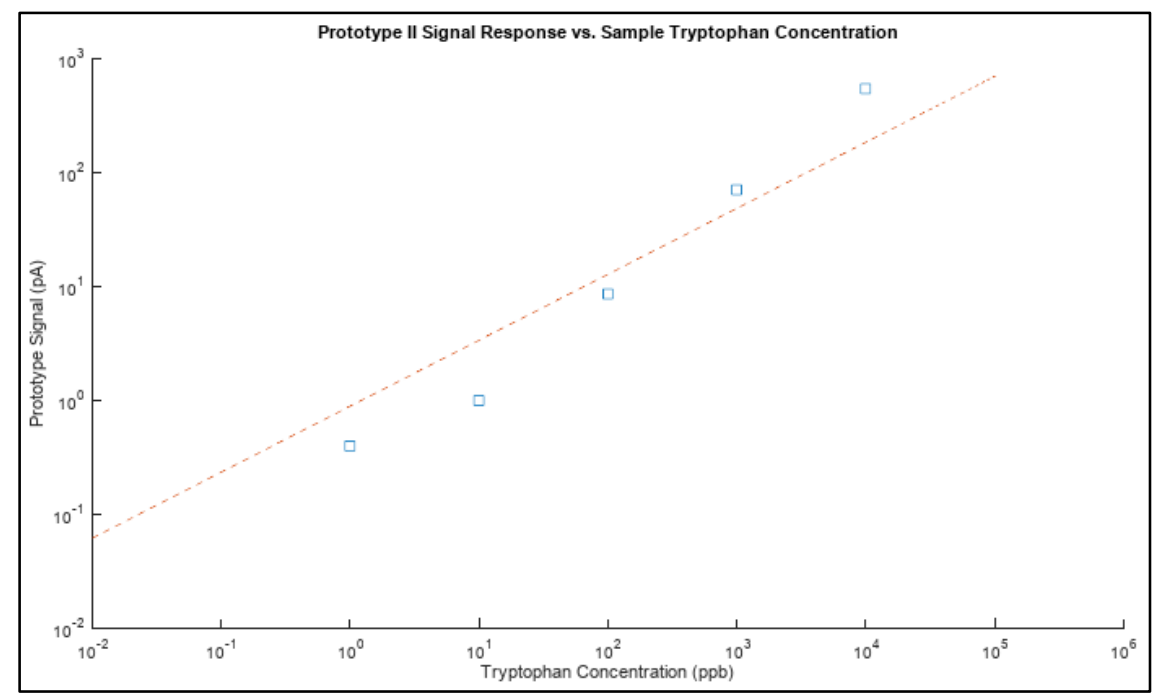

Figure 20: Linear Relationship between Tryptophan Concentration and Prototype II Response (data from D. Wilson, S. Croshere, personal communication, 2017)

The prototype shows a strong linear response to changes in tryptophan concentration, including dilutions at the low end of the expected sensitivity $\left(R^{2}=0.9991\right)$. Signals require significant amplification. 


\section{Section 5: Conclusions}

Given the overwhelming and unfulfilled global need for clean drinking water, and the high potential for contamination even in developed water systems, the adoption of an in-situ water sensor, deployed in an intelligent monitoring scheme, is a promising solution to an old and pressing problem. The viability of fluorescent analysis in the detection and even identification of indicator species has been established, and lowering costs of semiconductor technology make this choice all the more enticing.

This thesis contributes another comparison of TLF and TCC counts, finding a reasonable correlation in the case of city surface water in Portland, Oregon. In addition, an initial prototype for small residence times and a secondary optimization prototype designed to maximize native sensitivity have been explored.

\subsection{Summary}

Significant difficulties remain in the design process, including overcoming biofouling and mineral scaling on optical surfaces, maintaining and amplifying currents and voltages to both detectors and emitters, and developing novel analytical approaches to track contaminants in water distribution networks. But given the lack of alternative approaches offering high-temporal-resolution sensing technologies, fluorescent spectroscopy should be considered a promising solution to monitoring drinking water sources in response to contamination events. Currently, the literature shows strong correlations between the magnitude of TLF and the quantity of indicator species colonies within the TTC group, which is in line with the findings in this thesis. A second option is to set a safety threshold above which the TLF signal is taken to indicate biological 
contamination. With further research, fluorescent decay curves might be incorporated to identify specific bacterial species and improve the specificity of the method.

\subsection{Market for in-situ Fluorimeter}

Due to the significant global burden of disease presented by waterborne illnesses and the incompatibility of existing technologies with in-line sensing schemes, along with high up-front and consumables costs, the need for skilled labor and sterile environments, and the long wait times required between sampling and getting results using traditional water quality testing methods, the market is primed for products which use the robust correlation between TLF and TTC presence to serve as proxies for microbial contamination in drinking water sources. In addition to this capability, other fluorophores could be sensed using nearly-identical equipment, meaning that the product could be capable of providing valuable insights in a number of other fields, especially in the characterization of bodies of water in the environmental sciences, monitoring of drinking water for intentional or unintentional toxic contamination, and systematic testing of wastewater treatment products at various stages in the treatment process to decrease overall operating costs in terms of funds and energy consumption.

The high costs of existing fluorescent spectrometers can now be significantly offset by the use of semiconductor technology rather than expensive photomultipliers, along with the commercial availability of a large range of UV-compatible optical equipment, including bandpass filters, UV-transparent glass optics, and UV-transparent polymers. 


\subsection{Next Steps}

A second prototype has been developed which shows a linear response to very low concentrations of tryptophan. However, because the existing prototype requires a very powerful amplification circuit, noise in the signal becomes a significant challenge. It is therefore necessary to improve the sensitivity of the system prior to amplification to maintain a high signal quality for in-situ deployment.

Based on these original results, it appears initially unlikely that a photodiode's sensitivity will be compatible with UV polymer cuvettes given their high light attenuation, but because of the attractive costs and machinability of polymer parts, this option will still be explored before undertaking a design based on a flow-through quartz or silica cuvette.

A higher sensitivity will be achieved by experimenting with optical component placement, and perhaps with the addition of one to two collimating lenses and an additional converging lens. Another possible modification would be the use of a UV LED with a converging lens packaged at its head. The current prototype will allow for these experiments to be undertaken while holding all other components constant with machined precision. Once satisfactory sensitivity has been achieved, a set of in-situ fluorimeters will be manufactured and deployed, first under controlled lab conditions and then in the field. Sensitivity and specificity analyses will be generated based on field colony counts in order to examine the use of an in-situ fluorimeter as a microbial contamination monitor in low-resource settings. 


\section{Works Cited}

Albani, J. R. (2014). Origin of Tryptophan Fluorescence Lifetimes. Part 2: Fluorescence Lifetimes Origin of Tryptophan in Proteins. Journal of Fluorescence, 24, 105117.

Arana, I., Seco, C., Epelde, K., Muela, A., Fernandez-Astorga, A., \& Barcina, I. (2004). Relationships between Escherichia coli cells and the surrounding medium during survival processes. Antonie van Leeuwenhoek, 86, 189-199.

Bain, R., Bertram, J., Elliot, M., Matthews, R., McMahan, L., Tung, R., . . . Gundry, S. (2012). A Summary Catalogue of Microbial Drinking Water Tests for Low and Medium Resource Settings. International Journal of Environmental Research in Public Health, 9, 1609-1625.

Bain, R., Cronk, R., Wright, J., Yang, H., Slaymaker, T., \& Bartram, J. (2012). Fecal Contamination of Drinking-Water in Low- and Middle-Income Countries: A Systematic Review and Meta-Analysis. PLoS Med, 11(5), e1001644.

Baker, A., Cumberland, S. A., Bradley, C., Buckley, C., \& Bridgeman, J. (2015). To what extent can portable fluorescence spectroscopy be used in the real-time assessment of microbial water quality? Science of the Total Environment, 532, 14-19.

Baker, A., Ward, D., Lieten, S. H., Periera, R., Simpson, E. C., \& Slater, M. (2004). Measurement of protein-like fluorescence in river and waste water using a handheld spectrophotometer. Water Research, 38, 2934-2938.

Beer, K., Gargano, J. W., \& Roberts, V. A. (2015). Surveillance for waterborne disease outbreaks associated with drinking water--United States, 2011-2012. MMWR Morbidity \& Mortality Weekly Report, 64(31), 842-848.

Bharadwaj, R., Sai, V. V., Thakare, K., Dhawangale, A., Kundu, T., Titus, S., . . Mukherji, S. (2011). Evanescent wave absorbance based fiber optic biosensor for label-free detection of E. coli at $280 \mathrm{~nm}$ wavelength. Biosensors and Bioelectronics, 26, 3367-3370.

Bridgeman, J., Baker, A., Carliell-Marquet, C., \& Carstea, E. (2013). Determination fo changes in wastewater quality through a treatment works using fluroescent spectroscopy. Environmental Technology, 34(23), 3069-3077.

Brown, J., Stauber, C., Murphy, J. L., Khan, A., Mu, T., Elliot, M., \& Sobsey, M. D. (2011). Ambient-temperature incubation for the field detection of Escherichia coli in drinking water. Journal of Applied Microbiology, 110, 915-923.

Chang, K., Constantinescu, G., \& Park, S. (2006). Analysis of the flow and mass transfer 
processes for the incompressible flow past an open cavity with a laminar and a fully turbulent incoming boundary layer. Journal of Fluid Mechanics, 561, 113145.

Coble, P. G., Spencer, R. M., Baker, A., \& Reynolds, D. M. (2014). Aquatic Organic Matter Fluorescence. In P. G. Coble, J. Lead, R. M. Spencer, A. Baker, \& D. M. Reynolds, Aquatic Organic Matter Fluorescence (pp. 75-122). New York, New York, USA: Cambridge University Press.

Collaborators, G. 2. (2015). Global, regional, and national life expectancy, all-cause mortality, and cause-specific mortality for 249 causes of death, 1980-2015: a systematic analysis for the Global Burden of Disease Study 2015. The Lancet, 388(10053), 1459-1544.

Conmy, R., Del Castill, C., Downing, B., \& Chen, R. (2014). Chapter 6: Experimental Design and Quality Assurance: In Situ Fluorescence Instrumentation. In P. Coble, J. Lead, A. Baker, D. Reynolds, \& R. Spencer, Aquatic Organic Matter Fluorescence (pp. 190-230). New York: Cambridge University Press.

Cumberland, S., Bridgeman, J., Baker, A., Sterling, M., \& Ward, D. (2012). Fluorescences spectroscopy as a tool for determining microbial quality in potable water applications. Environmental Technology, 33(6), 687-693.

Dafour, A., \& Bartram, J. (2012). Animal waste, water quality and human health. London: IWA Publishing.

Dalterio, R. A., Nelson, W. H., Britt, D., Sprerry, J., Psaras, D., Tanguay, J. F., \& Suib, S. L. (1986). Steady-State and Decay Characteristics of Protein Tryptophan Fluorescence from Bacteria. Applied Spectroscopy, 40(1), 86-94.

Drost, K. J., Apte, S. V., Haggerty, R., \& Jackson, T. (2014). Parameterization of Mean Residence Times in Idealized Rectangular Dead Zones Representative of Natural Streams. Journal of Hydraulic Engineering, 140, 4014-4035.

Edberg, S. C., Rice, E. W., Karlin, R. J., \& Allen, M. J. (2000). Escherichia coli: the best biological drinking water indicator for public health protection. Journal of Applied Microbiology, 88(S1), 106S-116S.

Ferreira, A. P., Werneck, M. M., \& Ribeiro, R. M. (2001). Development of an evanescent-field fibre optic sensor for Escherichia coli O157:H7. Biosensors \& Bioelectronics, 16, 399-408.

Hambly, A., Henderson, R. K., Baker, A., Stuetz, R., \& Khan, S. J. (2010). Probabilistic analysis of fluorescence signals for monitoring dual reticulation water recycling schemes. Water Science \& Technology, 52(9), 2059-2065. 
Henderson, R. K., Baker, A., Murphy, K. R., Hambly, A., Stuetz, R. M., \& Khan, S. J. (2009). Fluorescence as a potential monitoring tool for recycled water systems: A review. Water Research, 43, 863-881.

Howard, G., Pedley, S., Barrett, M., Nalubega, M., \& Johal, K. (2003). Risk factors contributing to microbiological contamination of shallow groundwater in Kampala, Uganda. Water Resources, 37(14), 3421-3429.

Hudson, N., Baker, A., \& Reynolds, D. (2007). Fluorescence Analysis of Dissolved Organic Matter in Natural, Waste and Polluted Waters--A Review. River Resource Applications, 23, 631-649.

Hudson, N., Baker, A., \& Reynolds, D. (2007). Fluorescence Analysis of Dissolved Organic Matter in Natural, Waste and Polluted Waters--A Review. River Research and Applications, 23, 631-649.

Janke, R., Murray, R., Uber, J., \& Taxon, T. (2006). Comparison of Physical Sampling and Real-Time Monitoring Strategies for Designing a Contamination Warning System in a Drinking Water Distribution System. Journal of Water Resources Planning and Management, 132(4), 310-313.

Khamis, K., Sorensen, J. P., Bradley, C., Hannah, D. M., Lapworth, D. J., \& Stevens, R. (2015). In situ tryptophan-like fluorometers: assessing turbidity and temperature effects for freshwater applications. Environmental Science: Processes and Impacts, 17, 740-752.

Khush, R. S., Arnold, B. F., Srikanth, P., Sudharsanam, S., Ramaswamy, P., Durairaj, N., ... Colford, J. M. (2013). H2S as an Indicator of Water Supply Vulnerability and Health Risk in Low-Resource Settings: A Prospective Cohort Study. American Journal of Tropical Medicine Hygiene, 89(2), 251-259.

King, K. D., Vanniere, J. M., Leblanc, J. L., Bullock, K. E., \& Anderson, G. P. (2000). Automated Fiber Optic Biosensor for Multiplexed Immunoassays. Environmental Science \& Technology, 34, 2845-2850.

Lakowicz, J. R. (2006). Principles of Fluorescence Spectroscopy: Third Edition. New York, NY: Springer Science+Business Media, LLC.

Lee, H. H., Molla, M. N., Cantor, C. R., \& Collins, J. J. (2010). Bacterial charity work leads to population-wide resistance. Nature, $467,82-85$.

Lee, J.-H., \& Lee, J. (2010). Indole as an intercellular signal in microbial communities. FEMS Microbiological Review, 34, 426-444.

Liang, J. L., Dzuiban, E. J., Craun, E. J., Hill, V., Moore, M. R., Gelting, R. J., . . Roy, S. L. (2006). Surveillance for waterborne disease and outbreaks associated with 
drinking water and water not intended for drinking - United states, 2003-2004. Morbidity Mortality Weekly Reports, 55(SS12), 31-58.

Manja, K. S., Maurya, M. S., \& Rao, K. M. (1982). A simple field test for the detection of faecal pollution in drinking water. Bulletin of the World Health Organization, $60,797-801$.

Murray, R., Uber, J., \& Janke, R. (2005). A Model for Estimating the Acute Health Impacts resulting from Consumption of Contaminated Drinking Water. Impacts of Global Climate Change, 1-11.

Paruch, A. M., \& Maehlum, T. (2012). Specific features of Escherichia coli that distinguish it from coliform and thermotolerant coliform bacteria and define it as the most accurate indicator of faecal contamination in the environment. Ecological Indicators, 23, 140-142.

Sauer, M., Hofkens, J., \& Enderlein, J. (2011). Handbook of Fluorescence Spectroscopy and Imaging: From Single Molecules to Ensembles. Weinheim, Germany: Wiley$\mathrm{VCH}$.

Snoad, C., Nagel, C., Bhattacharya, A., \& Thomas, E. (2017). The Effectiveness of Sanitary Inspections as a Risk Assessment Tool for Thermotolerant Coliform Bacteria Contamination of Rural Drinking Water: A Review of Data from West Bengal, India . The American Journal of Tropical Medicine and Hygiene, 160322 .

Sorenson, J. R., Lapworth, D. J., Marchant, B. P., Nkhuwa, D. W., Pedley, S., Stuart, M. E., . . Chibesa, M. (2015). In-situ tryptophan-like fluorescence: A real-time indicator of faecal contamination in drinking water supplies. Water Research, 81, $38-46$.

Stedman, A. C., A., S. C., Seredynska-Sobecka, B., Boe-Hansen, R., Le Tallec, N., Waul, C. K., \& Arvin, E. (2011). A potential approach for monitoring drinking water quality from groundwater systems using organic matter fluorescence as an early warning for contamination events. Water Research, 45, 6030-6038.

Storey, M., van der Gaag, B., \& Burns, B. P. (2011). Advances in on-line drinking water quality monitoring and early warning systems. Water Research, 45, 741-747.

Tallon, P., Magajna, B., Lofranco, C., \& Leung, K. T. (2005). Microbial indicators of faecal contamination in water: a current perspective. 166(1), 139-166.

Tedetti, M., Joffre, P., \& Goutx, M. (2013). Development of a field-portable fluorometer based on deep ultraviolet LEDs for the detection of phenanthrene- and tryptophan-like compounds in natural waters. Sensors and Actuators B, 182, 416423. 
Valeur, B., \& Berberan-Santos, M. (2013). Molecular Fluorescence. Weinheim, Germany: Wiley-VCH.

Van Bergen, S. K., Bakaltcheva, I. B., Lundgren, J. S., \& Shriver-Lake, L. C. (2013). OnSite Detection of Explosives in Groundwater with a Fiber Optic Biosensor. Environmental Science \& Technology, 34, 704-708.

Wang, D., Ding, X., \& Rather, P. N. (2001). Indole Can Act as an Extracellular Signal in Escherichia coli. Journal of Bacteriology, 183(14), 4210-4216.

WHO. (2011). Guidelines for Drinking-water Quality. 4th ed. Geneva: WHO.

Wright, J. A., Yang, H., Walker, K., Pedley, S., Elliott, J., \& Gundry, S. W. (2012). The $\mathrm{H} 2 \mathrm{~S}$ test versus standard indicator bacteria tests for faecal contamination of water: systematic review and meta-analysis. Tropical Medicine and International Health, 17(1), 94-105.

Xu, J., Fischbeck, P. S., Small, M. J., VanBriesen, J. M., \& Casman, E. (2008). Identifying Sets of Key Nodes for Placing Sensors in Dynamic Water Distribution Networks. Journal of Water Resources Planning \& Management, 134(4), 378385 .

Yamashita, Y., \& Tanoue, E. (2003). Chemical characterization of protein-like fluorophores in DOM in relation to aromatic amino acids. Marine Chemistry, 82, 255-271.

Yang, H., Wright, J. A., Bain, R. E., Pedley, S., Elliot, J., \& Gundry, S. W. (2013). Accuracy of the H2S test: a systematic review of the influence of bacterial density and sample volume. Journal of Water and Health, 11(2), 173-185. 


\section{Appendix A: Membrane Filtration Water Testing Procedure}

1. If necessary, clean out the $44^{\circ} \mathrm{C}$ incubation chamber using DI water and dilute alcohol. Ensure that no MLSB buildup from previous tests is present in the incubation chamber.

2. Ensure that the incubator battery is charged, or that the incubator is connected to external power.

3. With a small amount of water in the test chamber, measure the temperature with a thermometer to ensure the chamber's heating elements are properly calibrated.

4. If the measured temperature is $+/$ - at least $0.5^{\circ} \mathrm{C}$ from the setpoint,

a. Calibrate using the calibration screw, letting the temperature stabilize for 30 minutes after each adjustment.

b. Turn the unit off and allow to cool for at least 12 hours.

c. Turn the unit back on and check the temperature to ensure calibration was successful.

5. Sterilize the filtration apparatus by burning with $1 \mathrm{ml}$ methanol, distributed evenly around the sampling cup. Once the flame has almost disappeared, invert the sampling chamber and place into the sampling cup to sterilize.

6. Using the DelAgua pad dispenser, dispense one pad into a sterilized petri dish.

7. Apply MLSB to the pad using a disposable pipette. The amount should be around $2.5 \mathrm{ml}$, and should cover all of the pad with slight excess showing around all edges.

8. Sterilize sample tweezers by applying the flame from a lighter to the connecting surfaces for about 5 seconds. 
9. Once again invert the filtration apparatus, and seal against the sample cup.

10. Open the sterile membrane filter packet and grasp the corner of the filter with the sterilized tweezers.

11. With one hand, loosen the filtration tube and lift above the apparatus; with the other hand, use sterilized tweezers to place a membrane filter on the top of the apparatus. Replace the filter funnel.

12. Tighten the collar of the filter funnel.

13. Invert the bottle containing the water sample twice to mix. Based on expected concentration of microbes, pour the sample into the funnel to the 10,50 or $100 \mathrm{ml}$ point.

14. Insert the pump bulb into the sample cup, and pump to pass the sample through the filter. Watch the sample carefully to observe any asymmetric behavior, which indicates the filter has been compromised.

15. Loosen the collar, and with one hand, remove the funnel from the filter apparatus. With the other hand, use the sterilized tweezers to remove the filter membrane from the apparatus.

16. Carefully place the filter membrane onto the prepared absorbent pad, using a gentle rolling motion to prevent the formation of air bubbles between. Observe the result as a quality check.

17. Place the lid on the petri dish, and make two records of the contents:

a. Mark the sample location and date on the petri dish.

b. Write down the sample location, date, and volume of water filtered in a separate set 
of logs, kept on paper and electronically.

18. Filter $100 \mathrm{~mL}$ of DI water through the filtration apparatus, using the vacuum pump.

19. Repeat steps 5-18 for each sample. Samples should be taken in groups of 2 from each location, with a DI blank and a site blank included for each location.

20. Allow all samples to sit for 30-60 minutes prior to incubation.

21. Clean and dry all parts of the filtration apparatus. Complete a methanol sterilization cycle for the filter cup and filtration apparatus before storage.

22. Stack the samples together using the petri dish carrier. Place the samples in the incubation chamber.

23. Incubate for 18 hours. 